\title{
Strain on ferroelectric thin films
}

\author{
Example of $\mathrm{Pb}\left(\mathrm{Zr}_{1-x} \mathrm{Ti}_{x}\right) \mathrm{O}_{3}$
}

\author{
Pierre-Eymeric Janolin
}

Received: 1 April 2009/Accepted: 5 May 2009/Published online: 2 June 2009

(C) The Author(s) 2009. This article is published with open access at Springerlink.com

\begin{abstract}
Strain engineering aims to take advantage of the stress field imposed by substrates on thin films. It requires an understanding of the consequences of stress fields on the physical properties of the deposited materials. This is achieved in ferroelectric thin films through the use of misfit-strain phase diagrams that show the stability regions for the possible phases. These encompass bulk phases as well as new ones exhibiting symmetries that are not present in the bulk. For the solid solution lead zirconate-lead titanate, $\mathrm{Pb}\left(\mathrm{Zr}_{1-x} \mathrm{Ti}_{x}\right) \mathrm{O}_{3}$, monoclinic phases found in the bulk morphotropic phase boundary region and associated to concentrations exhibiting the highest properties can be stabilized on a wider range of composition in thin films. In addition, phases of lower symmetry can be stabilized through the use of anisotropic biaxial stress fields, generated by orthorhombic substrates for example. Another crucial aspect of the influence of biaxial stress fields is the generation of domain structures. Theoretical tools as well as experimental verifications have provided much insight on the underlying physics. We, therefore, present here an overview of the influence of both iso- and anisotropic biaxial stress fields on the structures and properties of ferroelectric thin films exemplified on $\mathrm{Pb}\left(\mathrm{Zr}_{1-x} \mathrm{Ti}_{x}\right) \mathrm{O}_{3}$.
\end{abstract}

P.-E. Janolin $(\square)$

Laboratoire SPMS (Structures Properties and Modeling of Solids), CNRS-École Centrale Paris, Grande voie des Vignes, 92295 Châtenay-Malabry Cedex, France

e-mail: Pierre-Eymeric.Janolin@ecp.fr

\section{Introduction}

Ferroelectric thin films have attracted much attention for some years now, from both an application point of view and from a fundamental one. Applications-wise, their dielectric, piezoelectric, pyroelectric, and ferroelectric properties have stimulated many developments. From a fundamental point of view, both the 2D stress field as well as the effects linked to the reduced dimensionality have attracted much attention.

Many aspects of this field have been reviewed [1-4] and several books have been published on this topic [5-7]. In this article, we will focus on the thermodynamical description that has been given of the $\mathrm{Pb}\left(\mathrm{Zr}_{1-x} \mathrm{Ti}_{x}\right) \mathrm{O}_{3}(\mathrm{PZT}$ $1-x / x)$ solid solution deposited as thin films, on which most of the application are based.

The first part of this article will give a glimpse of the temperature-composition phase diagram of bulk PZT. Indeed, we shall see that the theoretical description of thin films compares their energies with respect to the bulk paraelectric one's. We shall also go briefly over the consequences of uniaxial stress and hydrostatic pressure on the properties of PZT. These stress fields, even though of another dimensionality compared to the biaxial one imposed on thin films, give some insight on the stressaccommodating mechanisms at play in this solid solution.

We shall then consider thin films restricting ourselves to epitaxial thin films, i.e., in which there is a preferential direction of growth. However, we shall consider the socalled "monodomain" as well as the "polydomain" cases, keeping in mind that "monodomain" (unless stated otherwise) in the tetragonal case stands here for thin films in which only $180^{\circ}$ domains exist whereas both $180^{\circ}$ and $90^{\circ}$ domains exist in a "polydomain" film. We shall then start by reviewing the theoretical tools used to predict whether 
thin films will be mono- or polydomain. Doing so, the prominent role played by thickness and dislocation density will appear.

We shall then focus on influence of isotropic biaxial stress field through the study of temperature-misfit strain phase diagrams for monodomain films, reviewing the hypothesis made in the calculations before describing their results for various compositions. We shall construct temperature-composition phase diagrams at fixed strain, in order to clarify the comparisons drawn with bulk PZT (temperature-composition) phase diagram.

Afterward, domain structures and temperature-misfit strain phase diagrams for polydomain PZT films will be reviewed. Comparison with the ones for monodomain thin films shall lead to some interesting conclusions about the possibility to stabilize a monoclinic phase in a PZT thin film.

These two descriptions will be followed by a discussion of the notion of misfit strain and its temperature evolution. Indeed, the misfit strain is far more complicated than the simple difference in lattice parameters between bulk PZT and the substrate. We shall demonstrate that the evolution of the misfit strain with temperature is neither linear nor vertical. This discussion shall be followed by an attempt to clarify the various notations used in the literature to describe the phases and the corresponding symmetries of both the polarization and the lattice.

In "Anisotropic 2D stress field" section, we shall study in the same way the influence of the change of symmetry in the stress field. In "Influence of thickness," section, we will focus on the influence of this crucial parameter and review the various critical thicknesses encountered in thin films. The "Future developments" section shall be devoted to some of the points that would potentially increase our ability to predict the structure as well as the properties of ferroelectric thin films. These developments should bring us closer to a fully predictive, realistic strain engineering of the properties of functional oxide thin films from which new miniaturized device will benefit.

As a final introductory note, we would like to emphasize that we would consider illusory to aim at completeness, considering the number of publications on this solid solution, and we sincerely apologize to the authors whose work would have been omitted in this article.

\section{Bulk PZT}

Temperature-composition phase diagram

In order to carry out a phenomenologically pertinent description of PZT thin films, prior knowledge of its bulk counterpart is mandatory. Bulk PZT temperature- composition phase diagram regained recently interest, requiring us to go briefly over some of the latest developments.

The main modification to the temperature-composition phase diagram established by Jaffe et al. in 1971 [8] on the basis of earlier works by Shirane and Takeda [9] was brought in the vicinity of the morphotropic phase boundary. This line was delimiting the rhombohedral zirconaterich side of the phase diagram and the tetragonal titanaterich one and had already been established to lie between PZT 60/40 and PZT 55/45 in 1952 [9]. Instead of a line, a boundary, a morphotropic phase region was established circa 2000. This region is characterized by monoclinic symmetries: $C m$ [10-13], $C c$ [14, 15], and $P m$ [16, 17], either alone or coexisting with the neighboring rhombohedral and tetragonal symmetries. These monoclinic phases are considered to act as a structural bridge between the rhombohedral and tetragonal sides of the phase diagram, enabling the rotation of the polarization within the monoclinic plane [18-20]. Noheda and Cox have reviewed recently this topic [21] and a model linking the local structure to the macroscopic symmetries was established [22]. The relation between the morphotropic region of PZT (among others) and piezoelectric properties was also reviewed recently by Bell [23]. The phenomenological implications of these monoclinic phases are that the expansion of the Landau polynomial has to be carried out to the twelfth order of the order parameter [24].

It has been recently reported that the actual symmetry of the zirconate-rich side of the diagram was monoclinic instead of rhombohedral [25]. The low-temperature rhombohedral phase would be $C c$ and the high-temperature one $\mathrm{Cm}$. The high piezoelectric response at the morphotropic phase boundary was then attributed to an elastic softening at the tetragonal to monoclinic phase transition [26]. These results have been reviewed with previous ones recently [27]. The nature of the monoclinic phase was also questioned through the consideration of the influence of nanodomains. It was showed through diffraction theory that rhombohedral nanodomains could produce reflections similar to the ones generated by a monoclinic symmetry [28, 29], contradicting this approach. The monoclinic symmetry of single domains as narrow as $30 \mathrm{~nm}$ in width was, however, found to be indeed monoclinic by convergent beam electron diffraction [30]. More research is still needed to clarify the exact nature of the temperaturecomposition phase diagram of bulk PZT, especially as it is often used as a model ferroelectric solid solution.

This morphotropic region is of primary interest as the dielectric constant, the piezoelectric and electromechanical coupling coefficients are maximized for these compositions or close to them. Therefore, either depositing thin films of the corresponding compositions or depositing thin films 
exhibiting the same structure are usual objectives for integrated device with optimized properties. In the bulk, the intrinsic width of this region was calculated to be 0.01 [31] and this width has been reported to be of 0.05 at $20 \mathrm{~K}$ [12] using solid-state chemistry. We will see that the composition range over which the monoclinic phases are stable in thin films may be much wider than in the bulk.

Very recently, the critical behavior of $\mathrm{Pb}\left(\mathrm{Zr}_{0.5} \mathrm{Ti}_{0.5}\right) \mathrm{O}_{3}$ has been studied by first-principles-based calculations and was found to belong to the 3d-random Ising universality class. This tends to suggest that, despite the much stronger long-range (dipolar) interactions, ferroelectrics adopt the same critical behaviors associated with universality classes as what is typically found in magnets [32]. This is of particular interest in the framework of multiferroics where ferroic orders are coupled, such as in the magnetoelectric effect, and for relaxors.

The end-member antiferroelectric $\mathrm{PbZrO}_{3}$ was less studied than $\mathrm{PbTiO}_{3}$. Nevertheless its dielectric [33-36], piezoelectric [37] and structural [38-40] properties as well as the ones with $<10 \%$ of $\mathrm{PbTiO}_{3}$ [9] were studied as early as the 50s. The existence of a ferroelectric rhombohedral phase on a narrow range of temperature (503-506 $\mathrm{K}$ upon heating and 505-500 $\mathrm{K}$ upon cooling) between the paraand antiferroelectric phases was confirmed later [41-43] and a detailed study of the phase transitions by combined $\mathrm{X}$-ray diffraction, dielectric and birefringence measurements determined the displacement of lead atoms (described by Kittel's theory of antiferroelectricity [44]) as well as the oxygen octahedra rotation [45]. The possibility to induce a ferroelectric phase thanks to (high) electric fields in the nominally antiferroelectric phase of $\mathrm{PbZrO}_{3}$ was reported and the electric field-temperature phase diagram for $\mathrm{PbZrO}_{3}$ established by Fesenko et al. [46]. The energetics and structural instabilities of lead zirconate have also been computed recently, underlying the small energy difference between the antiferroelectric and the ferroelectric phases [47] estimated quantitatively in the 50s [48].

\section{D and 3D stress fields}

If the deposition of a thin film on a substrate gives rise to a 2D stress field, study of $1 \mathrm{D}$ and 3D stress fields on PZT solid solution have also been carried out and brought into light stress-accommodating mechanisms that are at play in thin films.

Uniaxial pressure has been applied to zirconate-rich compounds [49, 50] and morphotropic compositions in order to study the reorientation of the domains [51]. Corresponding calculations have been carried out on monodomain lead titanate, phenomenologically [52] as well as by first-principles [53], pointing out the resulting enhancement of the piezoelectric coefficient along the polar axis. These experiments emphasize the fact that the least energetically costly mechanism for accommodating stress is domain wall movement and the consecutive reorganization of domains. If this reorganization is either not energetically favorable (for symmetry reasons for example) or if the stress is applied on a monodomain, it is then the symmetry of the lattice that accommodates the applied stress. A similar effect has been observed in other ferroelectric perovskites (see e.g. [54]), suggesting a common behavior under uniaxial pressure. We shall discuss the question of domain wall movement in thin films in relation to the structural defects in "Critical thickness for dislocations generation" section.

Hydrostatic pressure generates a 3D stress field. From lead zirconate [55-58] to lead titanate [59-63] through several intermediate compositions [64-68], PZT has been studied under pressure. Among the numerous interesting results in this field, a high-pressure form of ferroelectricity has been calculated and observed in $\mathrm{PbTiO}_{3}[59,60]$ at room temperature. At low temperature and still for $\mathrm{PbTiO}_{3}$, a monoclinic phase was calculated [69] and then observed [61], even though this last result and its implication have been questioned [63]. The rotation of the oxygen octahedra as a pressure-accommodating mechanism [70] is a common feature of the observations on lead titanate, as well as for $\mathrm{Pb}\left(\mathrm{Zr}_{1-x} \mathrm{Ti}_{x}\right) \mathrm{O}_{3}$. We will see that this stress-accommodation has not yet been taken into account in the theoretical descriptions of PZT thin films.

\section{Monodomain versus polydomain films}

\section{Domain phases}

The domain phases in bulk depend upon electrical and elastic boundary conditions. In thin films, these conditions are exacerbated due to the finite thickness and to the clamping onto the substrate.

If one considers an homogeneous polarization within a ferroelectric thin film, then the surfaces charges $(\sigma)$ accumulate on the interfaces: $\sigma=-\mathbf{n} \cdot \mathbf{P}$ with $\mathbf{n}$ the normal to the interface and $\mathbf{P}$ the polarization. These charges create a depolarizing field that counteracts the polarization, possibly suppressing it. In order to prevent the onset of the depolarizing field, the film may self-organize into ferroelectric domains, usually $180^{\circ}$ ones, or it may sustain such mono(ferroelectric)domain configuration by screening the surface charges thanks to metallic electrodes, therefore minimizing the depolarizing field.

Ferroelectric domains are created in bulk ferroelectric materials to reduce the electric field. In a similar manner, elastic domains coinciding with ferroelectric ones are created to minimize the elastic energy. The case of a 
domain wall between adjacent $90^{\circ}$ domains is illustrated on Fig. 1. We shall see that these modulated structures are formed as a consequence of phase transition rather than during the film growth.

From an X-ray diffraction point of view, (elastic) domain structures are sometimes not obvious to determine. In the monodomain tetragonal configuration, the lattice parameters are usually straightforward to determine (see Fig. 2) as there is only one out-of-plane and one in-plane lattice parameters. However, to determine precisely the out-of-plane lattice parameter, several (001) peaks have to be measured, in order to correct for any misalignment. The in-plane lattice parameter is deduced from the out-of-plane one and the measurement of several $(h 0 l)$ peaks, as in the tetragonal case $d_{h 0 l}=1 / \sqrt{h^{2} / a^{2}+l^{2} / c^{2}}$.

In the polydomain case however, the determination of the lattice parameters is slightly more complicated as the reciprocal lattices of several domain types superimpose. The case of two domains oriented at $90^{\circ}$ and having the same lattice parameters is illustrated on Fig. 3a. Note however that this is an approximation of the so-called $\mathrm{a} / \mathrm{c} / \mathrm{a} / \mathrm{c}$ domain structure where domains with polarization parallel to the growth direction (c domains) coexist with domains with polarization parallel to the interface with the

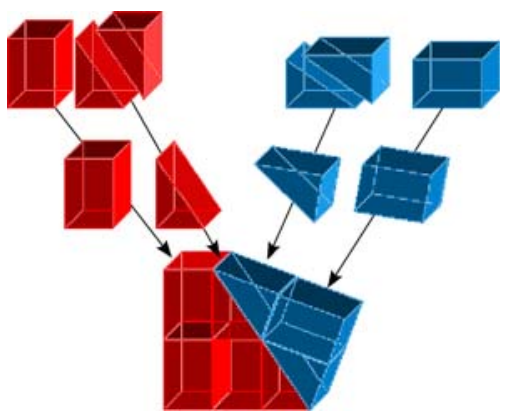

Fig. 1 Schematic representation of a domain wall between $90^{\circ}$ domains of tetragonal symmetry, exaggerating the tetragonality of the unit cell and therefore the misorientation resulting from this tetragonality. This is can be seen as the building block of an $\mathrm{a} / \mathrm{c} / \mathrm{a} / \mathrm{c}$ domain structure substrate (a domains). A detailed description of this structure shall be given in "Polydomain films" section.

The two out-of-plane lattice parameters will give rise to two peaks. This is illustrated on Fig. 3b. However, as the unit cell is not cubic, there is a slight misorientation of the two domain types (see Figs. 1 and 3c) that implies to correct the incidence angle (by $\Delta \omega^{\prime}$ ) in order to measure the maximum intensity from the peak. This is of particular importance if the domain population is of interest. Its determination is indeed based on the ratio of the integrated intensity of the peaks.

In order to determine the in-plane lattice parameters the same technique as for the monodomain case can be employed. However, in the present case reciprocal space maps (RSM) around the $(h 0 l)$ peaks (see Fig. 3d) are of particular interest. Indeed, in addition to the misorientation due to the tetragonality of the unit cell $\left(\Delta \omega^{\prime}\right)$, another difference in the incidence angle $(\Delta \omega)$ comes from the superposition of the reciprocal space maps of the two domain types, as illustrated on Fig. 3a. Therefore, RSM are a precious tool to precisely measure the positions of the peaks' maxima.

Various domain structures have been considered in firstprinciples calculations and Kornev et al. gave a review of these results recently [72]. This gives us an occasion to remind the reader that here, we consider domains from a structural point of view. Alternating ferroelectric $180^{\circ}$ domains, such as the stripes observed in [73], are composed of alternating tetragonal domains and are therefore labeled as monodomain here.

\section{Domain stability map}

In several PZT polydomain thin films deposited on $\mathrm{MgO}$ (i.e., on a cubic substrate with a larger lattice parameter), the volume fraction of the c domains in the film (hereafter $\alpha_{c}$ ) was found to be larger than that of a domains [74, 75]. For the special case of lead titanate deposited on $\mathrm{MgO}$ however, the situation is less clear and the observations differ significantly from one author to the other. The $\alpha_{c}$ 's were calculated in various ways, making the comparison
Fig. 2 a Reciprocal lattice and b X-ray diffraction pattern of a mono-domain $\mathrm{Pb}\left(\mathrm{Zr}_{0.2} \mathrm{Ti}_{0.8}\right) \mathrm{O}_{3}$ thin film on $\mathrm{SrTiO}_{3}$. The c domains give rise to only one out-of-plane lattice parameter, the corresponding diffraction peak is therefore single
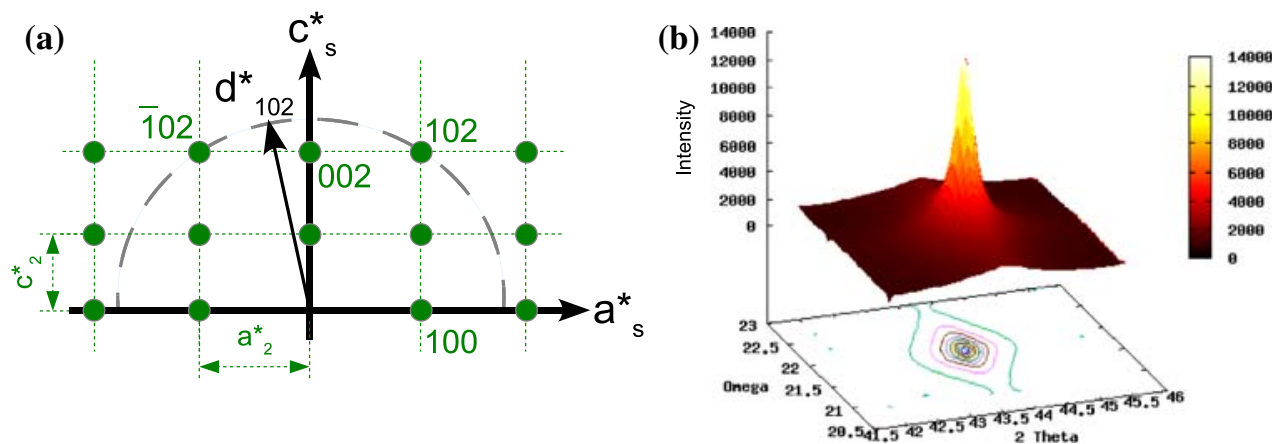
Fig. 3 a Reciprocal space and b XRD pattern of a poly-domain $\mathrm{Pb}\left(\mathrm{Zr}_{0.2} \mathrm{Ti}_{0.8}\right) \mathrm{O}_{3}$ thin film on $\mathrm{MgO}$. The $\mathrm{c}$ domains give rise to one out-of-plane lattice parameter and so do the a domains, there are therefore two diffraction peaks. The misorientation between the two domain types is illustrated in (c). The angle between the normals is sometimes reported as being $\pi / 2-2 \operatorname{arctg}(a / c)$ (e.g., in [71]). Though giving the exact same value for $\Delta \omega$, this expression takes the normal to the a domains as the reference, which seems less appropriate. The resulting difference in incidence $(\omega)$ angle between the two domain types as well as the separation in $2 \Theta$ is illustrated by the reciprocal space map on (d)
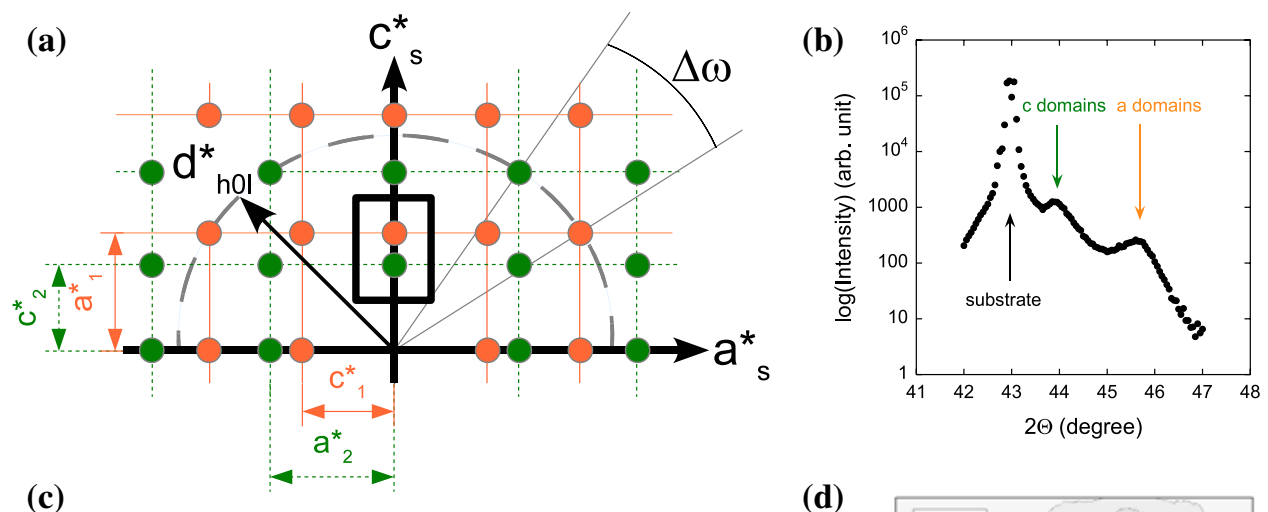

(c)

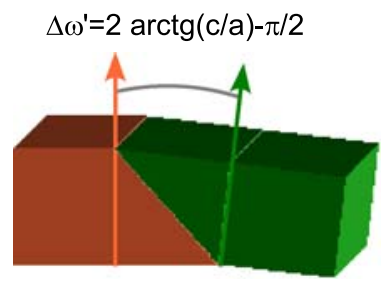

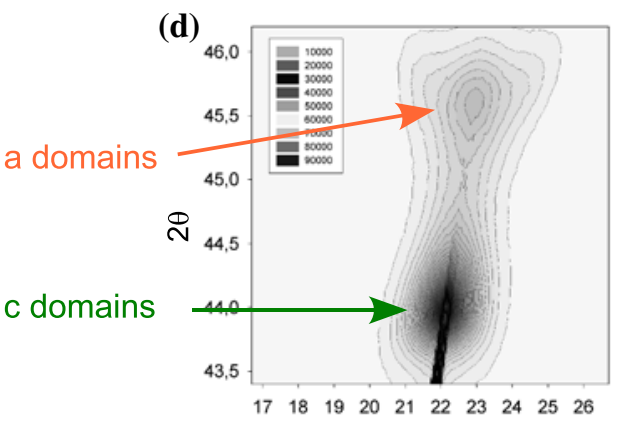

$\omega$ difficult (see [76] for a discussion of this problem). What is more, $\alpha_{c}$ depends on the deposition conditions, e.g. on the cooling pace [77] or on oxygen partial pressure for pulsed laser deposited films [78]. If an increase of $\alpha_{c}$ with thickness has been reported [79], most the experiments tend to show that $\alpha_{c}$ decreases with thickness [76, 80, 81]; On the contrary, its evolution with temperature makes a consensus: the decrease of $\alpha_{c}$ with temperature has been observed $[74,79,82,83]$ as well as calculated [84, 82].

Roytburd and Alpay, in a series of seminal papers [8587], have calculated domain stability maps that indicate the stable phase as a function of the tetragonality of the film and of the relative difference between the substrate and the film's in-plane lattice constants. These domain stability maps are a generalization of Pompe et al.'s domain pattern maps developed for twin domains [88] in the case of heterophase polydomain structures. The calculations were carried out in the short-circuit case (no depolarizing field) and were mainly considering a film much thicker than the critical thickness for domain formation (see "Critical thickness for dislocations generation" section). These calculations were later applied in the case when this approximation does not hold on the example of $\mathrm{Pb}\left(\mathrm{Zr}_{0.2} \mathrm{Ti}_{0.8}\right) \mathrm{O}_{3}$ [89] (see Fig. 4). These calculations consider a lamellar structure in which the period of the domain structure is smaller than the thickness of the film. This way, the domain wall energy is considered negligible compared to the energy within the domains and therein the polarization and strain fields may be regarded as homogeneous. Such a structure is expected in films thicker than $100 \mathrm{~nm}$ [90].

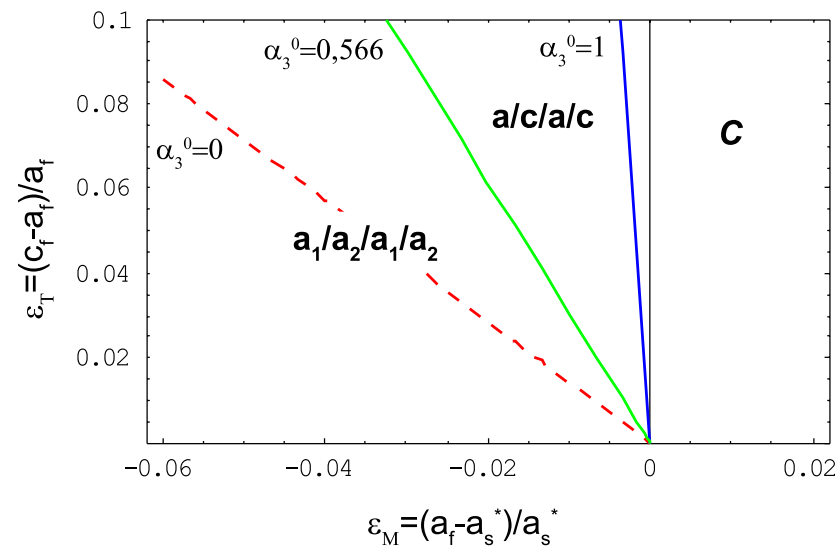

Fig. 4 Domain stability map for $\mathrm{Pb}\left(\mathrm{Zr}_{0.2} \mathrm{Ti}_{0.8}\right) \mathrm{O}_{3}$ as a function of the tetragonality of the film $\left(\varepsilon_{\mathrm{T}}\right)$ and misfit strain $\left(\varepsilon_{\mathrm{M}}\right)$. The thickness of the film is taken is 100 times larger than the critical thickness for domain formation. $\alpha_{3}^{0}$ stands for the equilibrium fraction of $\mathrm{c}$ domains. The full blue line separates the (mono) c domain phase $\left(\alpha_{3}^{0}=1\right)$ and the $\mathrm{a} / \mathrm{c} / \mathrm{a} / \mathrm{c}$ one $\left(0.566 \leq \alpha_{3}^{0} \leq 1\right)$; the green line separates the latter and the $a_{1} / a_{2} / a_{1} / a_{2}$ phase (see text). The $a / c / a / c$ phase is metastable for $0 \leq \alpha_{3}^{0} \leq 0.566$ and its limit of metastability is indicated by the red dashed line. The definition of the phases is given in the text

Therefore, there are four main points that can be drawn from these calculations are:

1. the only monodomain structure that can be stabilized by an isotropic biaxial strain is composed of $\mathrm{c}$ domains only.

2. A mono c domain structure can be stabilized under a modest biaxial tension if the tetragonality of the film is sufficient. This region is located between the vertical (black) line and the $\alpha_{\mathrm{c}}^{0}=1$ (blue) line. In addition, this 
region expands when the film's thickness decreases [89]. The stability region of the $\mathrm{a} / \mathrm{c} / \mathrm{a} / \mathrm{c}$ domain structure is also thickness dependent. For very thin films, the mono-c-domain phase transforms directly into an $a_{1} / a_{2} / a_{1} / a_{2}$ domain structure. The $a_{1} / a_{2} / a_{1} / a_{2}$ domain structure is characterized by domains oriented at $90^{\circ}$ from each other, the polarization therefore always points in the plane of the interface but either along [100] (for $a_{1}$ domains for example) or along [010] (for $\mathrm{a}_{2}$ domains then). It is worth noting that the domain walls are perpendicular to the interface.

3. $\alpha_{c}^{\mathrm{cr}}$ is defined as the proportion of $\mathrm{c}$ domains at which the $\mathrm{a} / \mathrm{c} / \mathrm{a} / \mathrm{c}$ domain structure becomes metastable. So when $\alpha_{c}<\alpha_{c}^{c r}$, the $a_{1} / a_{2} / a_{1} / a_{2}$ structure is stabilized at the thermodynamic equilibrium and hence $\alpha_{c}$ drops to zero. The evolution of $\alpha_{c}$ with temperature is therefore noncontinuous. For $\mathrm{Pb}\left(\mathrm{Zr}_{0.2} \mathrm{Ti}_{0.8}\right) \mathrm{O}_{3}$, thin films on $\mathrm{MgO}, \alpha_{\mathrm{c}}^{\mathrm{cr}}=0.566$. This means that the volume fraction of $\mathrm{c}$ domains in an $\mathrm{a} / \mathrm{c} / \mathrm{a} / \mathrm{c}$ domain structure will always be found larger than 0.566 if the thermodynamic equilibrium is reached. This is a consequence of the heterogeneous strain terms arising at the interface with the substrate. They counterbalance the elastic energy minimization that would favor the $\mathrm{a}_{1} / \mathrm{a}_{2} / \mathrm{a}_{1} / \mathrm{a}_{2}$ domain structure.

4. The volume fraction of $\mathrm{c}$ domains is a monotonous function of the tetragonality of the film $\left(\varepsilon_{\mathrm{T}}\right)$ as well as of the lattice mismatch $\left(\varepsilon_{M}\right)$.

The experimental evidence of the pertinence of the domain patterns maps developed by Pompe et al. [88] was brought by Foster et al. [91] with $\mathrm{PbTiO}_{3}$ thin films deposited on various substrates, and the domain stability map was also experimentally confirmed for tetragonal $\mathrm{Pb}\left(\mathrm{Zr}_{0.2} \mathrm{Ti}_{0.8}\right) \mathrm{O}_{3}$ thin films in [92] (where the hypothesis of short-circuit conditions or noninfluence of the depolarizing field was validated) and in [75].

\section{Isotropic biaxial stress field}

The influence of an isotropic 2D stress field imposed on films by substrates has been studied intensively as a function of temperature. Indeed, the application of such a strain field has dramatic consequences on the structure as well as on the properties of PZT thin films. This, however, is not necessarily true for all ferroelectrics, depending on the piezoelectric and elastic constants of the material [93]. The so-called temperature-misfit strain phase diagrams are a valuable tool for strain engineering. In addition, experimental measurements of shifts of transition temperatures and phase sequences as well as symmetries can be compared with theoretical predictions. For example, calculations have been carried out in the isotropic biaxial strain case by means of first-principles-based simulations and thermodynamics on several materials including $\mathrm{PbTiO}_{3}$ [94] and $\mathrm{PbZr}_{1-x} \mathrm{Ti}_{x} \mathrm{O}_{3}$ [95] considering the monodomain case as well as the polydomain one (respectively, in [96] and [90]).

There are only a few experimental determinations of the evolution of the misfit-strain with temperature [75, 97-99] whereas several evolutions of the complete set of lattice parameters evolution with temperature have been reported on $\mathrm{Pb}\left(\mathrm{Zr}_{1-x}, \mathrm{Ti}_{x}\right) \mathrm{O}_{3}$ thin films (e.g., see [100, 101] for monodomain films and [79, 82, 83, 102] for polydomain ones).

The misfit strain phase diagrams and the experimental evolution of the misfit strain for monodomain films are in good agreement for $\mathrm{PbTiO}_{3}$ thin films [98] as well as for $\mathrm{Pb}\left(\mathrm{Zr}_{0.2} \mathrm{Ti}_{0.8}\right) \mathrm{O}_{3}$ ones [97]. For polydomain films, the situation is not so clear as the calculation of the misfit strain does not always correspond to the one used theoretical papers [75, 99].

When the lattice parameters have been measured as a function of temperature, but the misfit strain has not been calculated, the comparison with the theoretical results are usually limited to the shift of the transition temperature.

Temperature-misfit strain phase diagrams for monodomain films

To calculate these phase diagrams in the monodomain case, one has to take into account the elastic energy induced during deposition and the consecutive strain because of the lattice mismatch but also the various coupling between strain and polarization. This implies that symmetries unstable in the bulk temperature-composition phase diagram may be stable in the temperature-misfit strain phase diagram. We will develop this point in the next paragraphs. Another consequence is the possible change of the order of the phase transition, as evidenced in $\mathrm{PbTiO}_{3}$ : the paraelectric to ferroelectric phase transition is of first order in the bulk and second order for thin films [94]. This is the consequence of the positive sign of the renormalized second-order coefficients $a_{i j}^{*}$, i.e., the ones multiplying the fourth power of the components of polarization, and not a consequence of a finite size effect [103].

The coordinate system used hereafter is the usual one (see Fig. 5): the $x, y$ and $z$ axes (sometimes labeled with subscripts 1,2 and 3) are linked to the $<100>$ directions of the substrate, with the $z$ (or 3 ) axis parallel to [001] substrate. The film is also supposed to be oriented so that $[100]_{\text {film }} / /$ $[100]_{\text {substrate }} / / x$ (or 1) and $[010]_{\text {film }} / /[010]_{\text {substrate }} / / y$ (or 2) and, consequently, for the growth direction: $[001]_{\text {film }} / /$ $[001]_{\text {substrate }} / / z$ (or 3). 


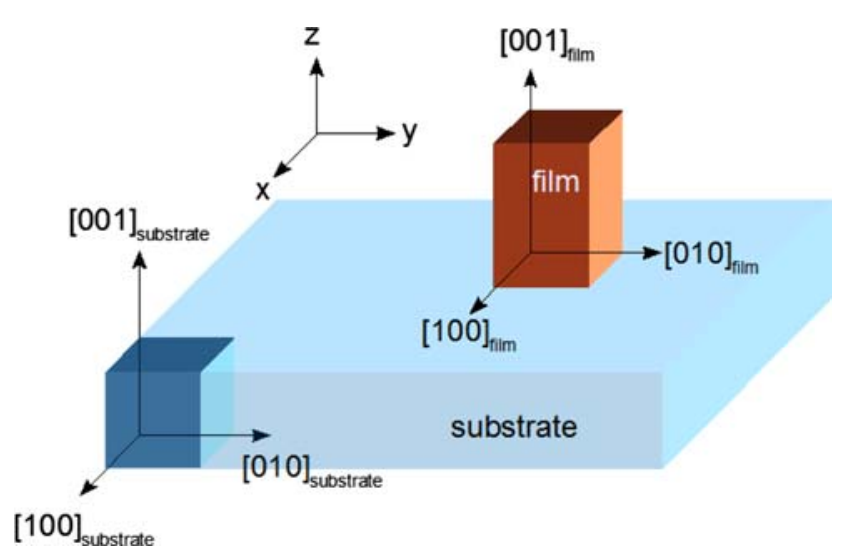

Fig. 5 Relative orientation of the substrate and film axes

A comprehensive review on first-principles modeling of ferroelectric thin films was recently published by Ghosez and Junquera [104] and we refer the reader to it and to the review by Kornev et al. [105] for a complete description of this field of intense research.

Phase field models usually consider both mono- and polydomain phases in their temperature-misfit strain phase diagrams and will therefore be mentioned in "Polydomain films" section.

We shall therefore restrict our consideration to the thermodynamical studies of monodomain ferroelectric thin film, starting by reviewing the main hypothesis that can be made in order to better understand the limitations as well as the possible improvements made.

\section{Hypothesis}

1. A fourfold symmetry at the surface is supposed, inducing an strain state with equal magnitude along two perpendicular directions of this surface. Such a strain state is often referred to as (biaxial) isotropic (misfit) strain state. That means either [001]-oriented tetragonal or cubic substrates are suited for such studies.

2. Coherent epitaxy, i.e., the in-plane lattice parameter of the films is equal to the one of the substrate.

3. The film is a pure $2 \mathrm{D}$ structure. There is no gradient of strain/polarization along the growth direction.

4. The substrate is not deformable, because of the ratio between its thickness and the ones of the film.

5. Perfect short-circuit electrical conditions. The space charges accumulation on the surface and at the interface are neglected.

6. The in-plane axes of the film are aligned with the ones of the substrate.

7. The surface of the substrate is a perfect plane.

\section{Limitations}

As any modeling, the temperature-misfit strain phase diagrams have limitations, some linked to the hypothesis, some implicit due to the Landau-Devonshire polynomial used.

The first limitation, linked with the biaxial symmetry of the strain state, was recently overcome and will be reviewed in "Anisotropic 2D stress field" section to consider the case where the films are deposited on orthorhombic substrates such as $\mathrm{NdGaO}_{3}$.

The concept of "effective" substrate relaxes the limitation linked to the coherent epitaxy [106]. Indeed, the growth of the film can still take place along the direction perpendicular to the interface, satisfying therefore the etymological meaning of epitaxy, but with dislocations at the interface. These dislocations reduce partially the elastic energy due to the deformation of the film's lattice. When the dislocation density is constant whatever the temperature, an "effective" substrate can be defined as the substrate effectively imposing the strain field on the film. The dislocations are thought to be created during deposition [107] and the temperature not to be high enough to enable further dislocation creation when the film is cooled from the deposition temperature down to room temperature. The critical thickness for dislocation generation will be considered in "Critical thickness for dislocations generation" section. It is worth noting that $180^{\circ}$ domain walls are perpendicular to the interface in the case of monodomain films. Indeed, in all the stable phases present on the temperature-misfit strain phase diagrams (c, $r$ and aa phases) the domain walls have no choice but to be perpendicular to the interface and not oriented at $\approx 45^{\circ}$ as in the case of a $90^{\circ}$ domain wall between $\mathrm{c}$ and a domains. This is indeed what has been observed [107-110] in thin films, even though the $101<101>$ slip system has often been considered, probably as Speck and Pompe mentioned it in their seminal paper [111], however cautiously indicating that this slip system was supposed to be the most common "based on a limited number of TEM studies," citing studies $[112,113]$ that were carried out on bulk perovskites presenting $90^{\circ}$ domains.

The effective substrate has the following characteristics:

- its lattice parameter $\left(a_{\mathrm{s}}^{*}\right)$ is linked to one of the real substrate $\left(a_{\mathrm{s}}\right)$ through the density of dislocation $(\rho)$ by: $a_{\mathrm{s}}^{*}(T)=\frac{a_{\mathrm{s}}(T)}{1 \pm \rho a_{\mathrm{s}}(T)}$. We added the \pm sign to the original expression [87]: the $+(-)$ sign standing for the case when $a_{\mathrm{s}}>a_{\mathrm{s}}^{*}>a_{\mathrm{b}}\left(a_{\mathrm{s}}<a_{\mathrm{s}}^{*}<a_{\mathrm{b}}\right)$ with $a_{\mathrm{b}}$ the lattice parameter of the bulk at the deposition temperature.

- its thermal expansion coefficient $\left(\alpha_{\mathrm{s}}^{*}\right)$ is proportional to the one of the real substrate $\left(\alpha_{\mathrm{s}}\right)$ through: $\alpha_{\mathrm{s}}^{*}=\frac{a_{\mathrm{s}}}{a_{\mathrm{s}}^{*}} \alpha_{\mathrm{s}}$. Thermal evolutions of $a_{\mathrm{s}}^{*}$ and $a_{\mathrm{s}}$ only then are parallel. 
- the thermodynamics calculations carried out in the framework of coherent epitaxy can be used, simply replacing $a_{\mathrm{s}}$ by $a_{\mathrm{s}}^{*}$.

The vast majority of the experimental reports on monodomain films indicate that the in-plane axis are indeed aligned with the substrate's ones. This is generally checked through a $\phi$-scan carried out on a $(h 0 l)$ plane. This consists in selecting a diffraction peak corresponding to a plane that is not parallel to the interface and to turn the sample around the normal to the growth direction (i.e., around the angle usually called $\phi$ ). One then gets four peaks, separated by $\pi / 2$, corresponding to the fourfold symmetry of the substrate and a set of four other peaks, at the same $\phi$ values, equally spaced over $2 \pi$. In the case of $\mathrm{PbTiO}_{3}$ deposited on $\mathrm{MgO}$, another orientation is possible [80]. Indeed the film may be rotated by $\pi / 4$ with respect to the substrate's axes, in order to minimize the elastic strain due to the lattice mismatch. $\mathrm{MgO}$ has indeed a lattice parameter much larger than that of bulk PZT.

The perfect nature of the substrate is aimed experimentally, through appropriate chemical preparations (see for example [114, 115] and references therein for $\mathrm{SrTiO}_{3}$ ), as it was showed that a vicinal substrate could be used to control the (poly)domain structure [116].

There is another implicit limitation in the calculations as some terms as the ones coupling the stress to the fourth power of the polarization are absent, the corresponding coefficients being not available in the literature to the best of our knowledge.

\section{Temperature-composition phase diagrams at constant misfit}

The temperature-misfit strain phase diagrams for monodomain $\mathrm{Pb}\left(\mathrm{Zr}_{1-x} \mathrm{Ti}_{x}\right) \mathrm{O}_{3}$ over the whole composition range $[117,118]$ or for $0.4 \leq x \leq 0.9$ [95] as well as $x=1.0$ [94] have been calculated. In order to visualize the influence of strains on the temperature-composition phase diagram, three strains have been chosen, negative $\left(s_{\mathrm{m}}=-0.4 \%\right)$, zero $\left(s_{\mathrm{m}}=0\right)$, and positive $\left(s_{\mathrm{m}}=+0.8 \%\right)$ in order to illustrate these changes.

At negative misfit strain Figure 6 illustrates the case of a negative misfit strain (i.e., under compression with respect to the unstrained cubic parent phase). The monoclinic $r$ phase is stable for $\mathrm{Ti}$ concentrations lower than $60 \%$ and the highest temperature of stability of this monoclinic phase is in the vincinity of $x=0.5$.

This temperature-composition phase diagram is qualitatively different from the ones calculated by $\mathrm{Oh}$ et al. $[117,118]$, probably because the monoclinic phase defined by $\mathrm{P}_{1}=\mathrm{P}_{2} \neq 0, \mathrm{P}_{3} \neq 0$ by Pertsev et al. [94] is only

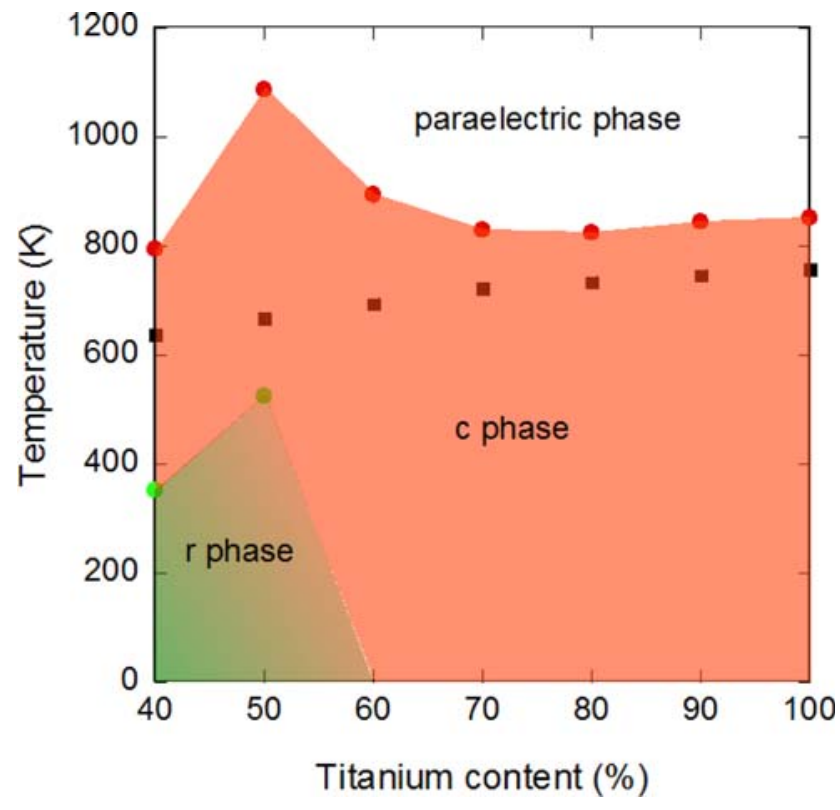

Fig. 6 Temperature-composition phase diagram for $\mathrm{Pb}\left(\mathrm{Zr}_{1-x} \mathrm{Ti}_{x}\right) \mathrm{O}_{3}$ $(x \geq 0.4)$ for constant biaxial isotropic misfit strain of $-0.4 \%$ (see Table 1 for details about the phase symmetries). The black squares indicate the bulk para-ferroelectric transition temperatures for comparison purpose. Points extracted from [95]

considered by Oh et al. $[117,118]$ in the special case where $\mathrm{P}_{1}=\mathrm{P}_{2}=\mathrm{P}_{3}$. Therefore, this monoclinic phase does not appear in their temperature-composition phase diagrams whereas the condition $\mathrm{P}_{1}=\mathrm{P}_{2}=\mathrm{P}_{3}$ is mentioned in Pertsev's temperature-misfit strain phase diagrams by a doted line [95]. It is worth noting that labeling as orthorhombic $[117,118]$ a phase defined by $\mathrm{P}_{1}=\mathrm{P}_{2}=\mathrm{P}_{3}$ is inappropriate. We shall come back to this point in the "Clarification of the notations used in the isotropic biaxial stress field case" section.

The ferro- to paraelectric transition temperatures are not monotonous with composition on the contrary to the bulk. This is due to the fact that the increase in the para- to ferroelectric transition temperature with respect to the bulk is proportional to [94] $\frac{C \cdot Q_{i j}}{s_{11}+s_{12}} \cdot s_{\mathrm{m}}$ with $C$ the Curie-Weiss constant, $Q_{i j}$ electrostrictive coefficients $\left(Q_{i j}=2 \cdot Q_{12}\right.$ or $Q_{i j}=Q_{11}+Q_{12}$ for a transition to the tetragonal ferroelectric c phase and to the orthorhombic aa phase, respectively), $s_{i j}$ the elastic compliances and $s_{m}$ the misfit strain.

At zero misfit strain Figure 7 illustrates the influence of clamping a ferroelectric material on a substrate. Indeed, the temperature-composition phase diagram for thin films at zero misfit strain exhibits two phases: a monoclinic $r$ phase for $\mathrm{Ti}$ concentration smaller than $70 \%$ and a tetragonal $\mathrm{c}$ phase for Ti concentration larger than $60 \%$. This is a major difference with the bulk. The ferro to paraelectric transition 


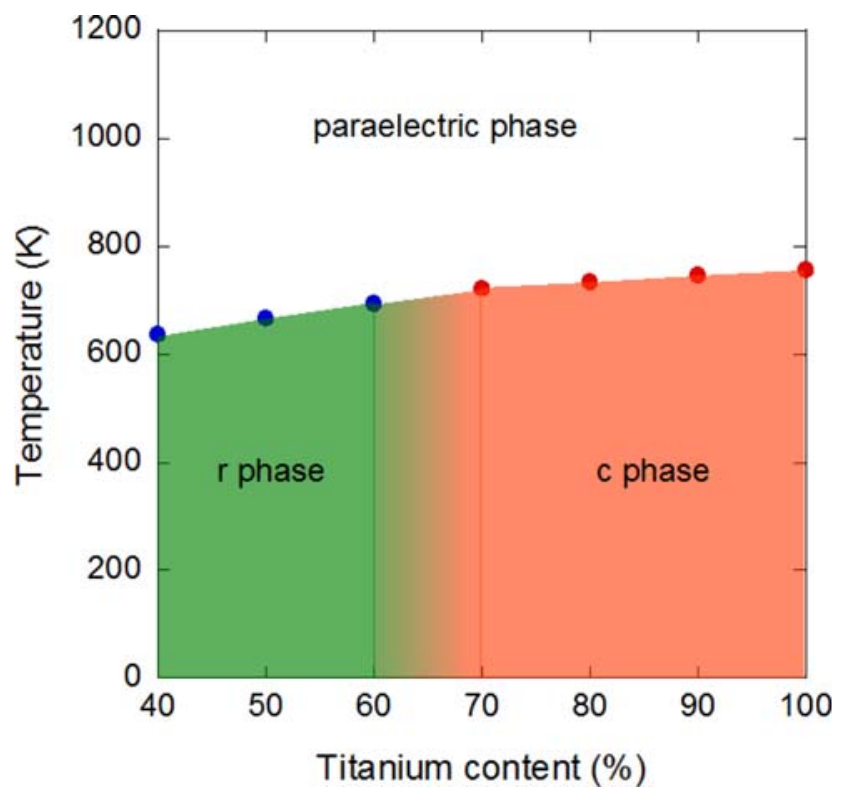

Fig. 7 temperature-composition phase diagram for $\mathrm{Pb}\left(\mathrm{Zr}_{1-x} \mathrm{Ti}_{x}\right) \mathrm{O}_{3}$ $(x>0.4)$ for zero biaxial isotropic misfit strain. The para-ferroelectric transition temperatures are equal to the bulk ones, for comparison purposes. Points extracted from [95]

temperatures are however the same as in the bulk and the monotonous evolution with concentration of this temperature observed for the bulk remains in thin films. This is due to the fact that the increase in the ferro to paraelectric phase transition temperature is a monotonous function of the misfit strain (see Eq. 4 in [94]). If the misfit strain is zero the temperatures at which the paraelectric phase loses its stability with respect to both the tetragonal and the monoclinic phases are then equal to the Curie-Weiss temperature of the bulk.

The question then arises of the reason of this discrepancy between the symmetries of the ferroelectric phases in the film and in the bulk at zero misfit strain. The discrepancy lies in the definition of the misfit strain as the difference between the strained state of the system (i.e., its in-plane lattice parameter) and the thermodynamical reference unstrained free state (i.e., the pseudocubic lattice parameter of the bulk). This implies that a zero misfit strain corresponds to the bulk only in the paraelectric case. Therefore, there is no reason why the ferroelectric phases should adopt the same symmetry as the bulk ones. We shall develop this point in the "Misfit strain" section.

At positive misfit strain In the positive case $\left(s_{\mathrm{m}}=\right.$ $+0.8 \%$ ) displayed in Fig. 8, there is a low temperature monoclinic $r$ phase throughout the considered composition range (i.e., for $\mathrm{Ti}$ concentration greater than $40 \%$ ) above which lies the orthorhombic so-called aa phase that eventually transforms into the tetragonal paraelectric phase

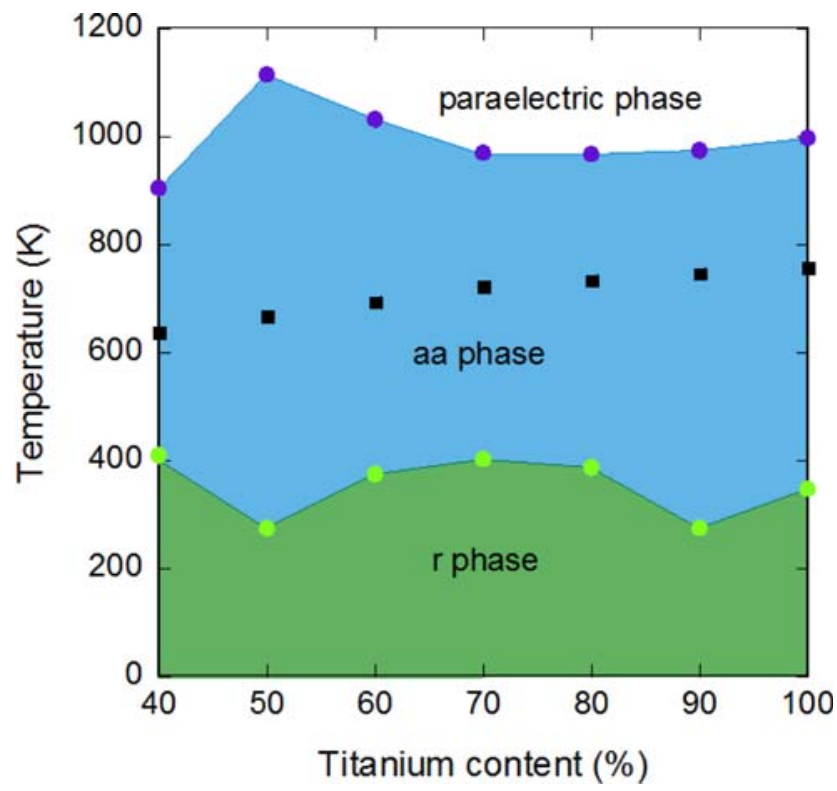

Fig. 8 Temperature-composition phase diagram for $\mathrm{Pb}\left(\mathrm{Zr}_{1-x} \mathrm{Ti}_{x}\right) \mathrm{O}_{3}$ $(x \geq 0.4)$ for constant biaxial isotropic misfit strain of $+0.8 \%$. The black squares indicate the bulk para-ferroelectric transition temperatures, for comparison purposes. Points extracted from [95]

upon heating. As for the negative case $\left(s_{\mathrm{m}}=-0.4 \%\right.$, Fig. 6), the evolution of the Curie temperature with composition is not monotonous, for the same reason.

As a conclusion, the strain effect is more subtle than a simple shift [117-119] of the temperature-composition phase diagram of bulk PZT and enable the stabilization of monoclinic phases on a much wider range of compositions than in the bulk.

Polydomain films

\section{Domain structure}

The possible domain structures on a cubic substrate have been labeled:

- c (monodomain) structure, when the polarization is pointing perpendicularly at the interface. These domains are tetragonal with a symmetry $P 4 \mathrm{~mm}$.

- $a_{1} / a_{2} / a_{1} / a_{2}$ domain structure. $a_{1}$ domains have their polarization pointing along the direction $x$ (or 1) of the substrate (see Fig. 5) whereas $a_{2}$ domains have their polarization pointing along the perpendicular in-plane direction. These domains are orthorhombic. Indeed, the displacement of the B cation along [100] or [010] breaks the fourfold symmetry and induces the orthorhombic symmetry. A structure composed of a single type of a domains is however always less stable than a structure in which both type of a domains coexist [87]. This changes when an anisotropic strain is imposed on 
the film by means of orthorhombic substrates (see "Anisotropic 2D stress field" section).

- $\quad \mathrm{c} / \mathrm{a} / \mathrm{c} / \mathrm{a}$ domain structure. In this structure $\mathrm{c}$ and one type of a domains coexist. As a consequence, this structure could be labeled $\mathrm{c} / \mathrm{a}_{i} / \mathrm{c} / \mathrm{a}_{i} \quad(i=1,2)$. In this structure, $\mathrm{c}$ domains are tetragonal and a domains orthorhombic (the lattice parameters for such a phase are reported in Fig. 11).

- a structure in which the three domains coexist is theoretically possible and would consist of the coexistence of $\mathrm{c} / \mathrm{a}_{1} / \mathrm{c} / \mathrm{a}_{1}$ and $\mathrm{c} / \mathrm{a}_{2} / \mathrm{c} / \mathrm{a}_{2}$ domain structures. However, it has been shown [120] that symmetry imposes the volume fraction of $c$ domains to be equal in both structures and the volume fraction of $c / a_{1}$ and $c / a_{2}$ to be also equal. The equilibrium elastic energy corresponds to a volume fraction of c domains of $1-$ $2 \frac{a_{\mathrm{s}}-a_{\mathrm{b}}}{c_{\mathrm{b}}-a_{\mathrm{b}}}$ with $a_{\mathrm{b}}, \quad c_{\mathrm{b}}$ the lattice parameters of the tetragonal bulk and $a_{\mathrm{s}}$ the substrate's lattice parameter. The substrate's lattice parameter then has to satisfy: $a_{\mathrm{s}}<\frac{a+c}{2}$

- a $r^{*} / a a^{*} / r^{*} / a a^{*}$ structure is a polydomain structure where the $r^{*}$ domains $\left(\mathrm{P}_{1} \neq 0, \mathrm{P}_{2} \neq 0, \mathrm{P}_{3} \neq 0\right.$, and $\mathrm{P}_{1} \simeq \mathrm{P}_{2}>>\mathrm{P}_{3} \neq 0$ at large misfits) coexist with the $a a^{*}\left(\mathrm{P}_{1}, \mathrm{P}_{2}>>\mathrm{P}_{3} \neq 0\right)$ ones.

The possibility of having several layers with different domain structures on top of each other has not been specifically addressed theoretically even though this possibility was reported for tetragonal PZT thin films [121]. In addition, the case of 3-domain structures has only been considered under stress-free conditions to explain experimental results [122].

\section{Temperature-misfit strain phase diagrams for polydomain films}

Temperature-misfit strain phase diagram considering polydomain structures, when calculated from thermodynamical considerations, are based on the monodomain ones. The relative stability of the polydomain phases is compared to the monodomain ones. This methodology has been applied to $\mathrm{PbTiO}_{3}$ (and $\mathrm{BaTiO}_{3}$ ) by Pertsev et al. [123]. The considered system consists of thin films with a periodicity of the lamellar domain structure much smaller than the thickness of the film, under short-circuit electric boundary conditions. In these films, the depolarizing field is negligible and does not affect the domain structure ([123] and references therein).

The equilibrium fraction of $\mathrm{c}$ domains $\left(\phi_{\mathrm{c}}^{*}\right)$ is given by (Eq. 2, [123]): $\phi_{\mathrm{c}}^{*}=1-\frac{\left(s_{11}-s_{12}\right)\left(s_{\mathrm{m}}-Q_{12} P_{\mathrm{s}}^{2}\right)}{s_{11}\left(Q_{11}-Q_{12}\right) P_{\mathrm{s}}^{2}}$ with $s_{i j}$ the elastic compliances, $Q_{i j}$ the electrostrictive coefficients, $P_{\mathrm{s}}$ the spontaneous polarization (which implies naturally that the domain structure is only stable in the ferroelectric phases, where $P_{\mathrm{s}} \neq 0$ ), and $s_{\mathrm{m}}$ the misfit strain. This expression implies a monotonous evolution of $\phi_{c}^{*}$ with the misfit strain, in agreement with the domain stability maps evoked in "Domain stability map" section.

The main consequence of the taking into account of the polydomain structures is to destabilize the monodomain monoclinic $r$ phase. This is bad news from an application point of view as stabilizing such a phase in which the polarization is free to rotate is supposed to be the key to high properties in thin film. It can also be considered as an additional key issue for strain engineering, namely controlling the parameters determining whether the polydomain state or the monodomain one will be stable under realistic conditions. The related influence of thickness will be addressed in "Critical thickness for mono- to polydomain transition" section.

\section{Discussion}

\section{Misfit strain}

As we have mentioned before, the misfit strain is defined as the relative difference between the lattice parameter of the constrained system (the film) and its lattice parameter in the reference unstrained (pseudo)cubic paraelectric phase. It is the sum of three terms: the elastic strain imposed by the lattice difference between the film and the substrate, the thermal strain as the substrate imposes its thermal evolution onto the film, and the spontaneous transformation strain, which is linked to the phase transitions the bulk material undergoes.

The thermal strain, however, is not necessarily the main parameter to consider when performing strain engineering, as the mismatch in lattice parameters at the deposition temperature determines the initial misfit strain. The stress corresponding to this initial stage of the misfit strain (in other words the deposition strain, lattice mismatchinduced) can be as high as a few GPa [89]. This initial misfit strain or deposition strain can be influenced by the deposition conditions. For example, oxygen vacancies tend to increase the lattice parameters of perovskites, as it has been shown for $\mathrm{BaPbO}_{3}$ [124] or $\mathrm{SrTiO}_{3}$ [125]. For the latter, it has also been shown that the transition temperature between the antiferrodistortive and cubic phases is reduced by the oxygen vacancy concentrations [125, 126]. An original way to engineer this strain is to bend the substrate during deposition and to let it straighten back afterward, therefore inducing an additional static strain [127].

In the case of thin films grown coherently with the substrate (i.e., when films adopt the substrate's lattice parameter) this corresponds to $s_{\mathrm{m}}=\frac{b-a_{0}}{a_{0}}$, with $b$ the 
substrate's lattice constant and $a_{0}$ the pseudocubic bulk lattice parameter. This latter is equal to the cubic lattice parameter (for $T>T_{\mathrm{C}}^{\text {bulk}}$ ) in the paraelectric phase and to its linear extrapolation in the ferroelectric phase (for $T<$ $\left.T_{C}^{\text {bulk }}\right)$. This definition is slightly different from the one proposed by Pertsev et al. [94] as we used $a_{0}$ at the denominator instead of $b$ in order to have a definition that would illustrate the strain between the final state (corresponding to $b$ ) and the initial (reference) state (corresponding to $a_{0}$ ). However, $b-a_{0}<<b$ and $a_{0}$ allows the comparison.

If the growth is incoherent, i.e., if misfit dislocations at the interface relax part of the strain imposed on the film, then the lattice constant of the real substrate $(b)$ should be replaced by the one of the effective substrate $\left(b^{*}\right)$ [111] evoked in "Limitations" section. Naturally, $b^{*}$ goes from the substrate's lattice parameter $b$ when no dislocations are present to the lattice parameter of the bulk (i.e., a or c for tetragonal compositions) when the dislocations relax completely the strain. But going further, this effective substrate is defined as the one actually imposing the misfit strain on the film, in other words it acts as the substrate on which the film would be in coherent epitaxy, it is then straightforward to see that $b^{*}=a_{/ /}$, with $a_{/ /}$the in-plane lattice parameter of the film.

Figure 9 shows the thermal evolution of a $\mathrm{Pb}\left(\mathrm{Zr}_{0.2}\right.$ $\left.\mathrm{Ti}_{0.8}\right) \mathrm{O}_{3}$ thin film deposited on $\mathrm{SrTiO}_{3}$. The deposition $\left(\varepsilon^{\text {deposition }}\right)$, thermal $\left(\varepsilon^{\Delta \text { th }}\right)$, and spontaneous $\left(\varepsilon^{\text {spont }}\right)$ strains are reported. The deposition strain has already been mentioned, let us now turn to the deposition strain.

The thermal evolution of the misfit strain in the general case where dislocations are present at the interface is given by:

$s_{\mathrm{m}}(T)=\frac{a_{/ /}\left(T_{0}\right)\left(1+\alpha_{b^{*}} \cdot \Delta T\right)-a_{0}\left(T_{0}\right)\left(1+\alpha_{a_{0}} \cdot \Delta T\right)}{a_{0}\left(T_{0}\right)\left(1+\alpha_{a_{0}} \cdot \Delta T\right)}$

with $\alpha_{b^{*}}$ and $\alpha_{a_{0}}$ the thermal expansion coefficients (in $\mathrm{K}^{-1}$ ) of the effective substrate and of the pseudocubic bulk, respectively, and $\Delta T=T-T_{0}$ the difference between the considered temperature and room temperature. The thermal expansion coefficients are taken independent of the temperature to simplify the notations; this approximation is generally valid between deposition and room temperatures but not so much below $100 \mathrm{~K}$. What is more, taking into account the dependence of the thermal expansion coefficient upon temperature would strengthen our conclusion. Let us repeat that $\alpha_{b^{*}}$ is not the thermal expansion coefficient of the real substrate $\left(\alpha_{b}\right)$ and that it is proportional to it: $\alpha_{b^{*}}=\frac{b}{b^{*}} \cdot \alpha_{b}$. They are only equal when no dislocations are present $(\rho=0)$, however, $\alpha_{b^{*}}$ is not equal to $\alpha_{a_{0}}$ when the strain is fully relaxed $\left(\rho=\rho_{\max }\right)$.

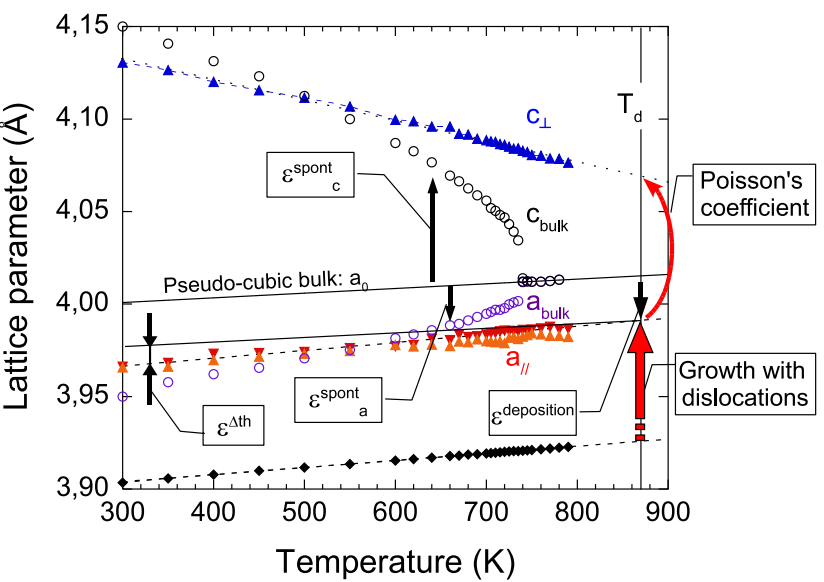

Fig. 9 Evolution with temperature of the in- $\left(\mathrm{a}_{/ /}\right.$, orange and red triangles $)$ and out-of-plane $\left(\mathrm{c}_{\perp}\right.$, blue triangles) lattice parameters of $\mathrm{Pb}\left(\mathrm{Zr}_{0.2} \mathrm{Ti}_{0.8}\right) \mathrm{O}_{3}$ thin film deposited on $\mathrm{SrTiO}_{3}$ (b, black diamonds), compared with the one of tetragonal bulk $\mathrm{Pb}\left(\mathrm{Zr}_{0.2} \mathrm{Ti}_{0.8}\right) \mathrm{O}_{3}$ (empty circles: $\mathrm{c}$ (black) and a (purple)). Misfit dislocations are present at the interface as $a_{/ /} \neq b$, relaxing partially the deposition strain ( $\left.\varepsilon^{\text {deposition }}\right)$ at the deposition temperature $\left(T_{\mathrm{d}}\right)$. This strain is transfered through the Poisson's coefficient to $c_{\perp}$. It adds to the ferroelastic strain as the film is already ferroelectric. When cooled through $T_{\mathrm{C}}^{\text {bulk }}$, a spontaneous strain $\left(\varepsilon^{\text {spont }}\right)$ appears, both for the a and $\mathrm{c}$ lattice parameters. Under $T_{\mathrm{C}}^{\text {bulk }}$, the pseudocubic bulk lattice parameter $\left(a_{0}\right)$ becomes different from the bulk lattice parameters and keeps a linear evolution represented by a full line. The second full line underneath starts from the extrapolated value $a_{/ /}\left(T_{\mathrm{d}}\right)$ and diverges from the doted line that stands for the thermal evolution of the effective substrate $b^{*}(T)$. This divergence represents the thermal strain $\left(\varepsilon^{\Delta \text { th }}\right)$ that develops into the film. The misfit strain is the sum of these three strains and is calculated by the relative difference between $a_{/ /}$and $a_{0}$

This is not surprising as we have considered a constant density of misfit dislocation with temperature. This is evidenced by the parallel thermal evolutions of the in-plane lattice parameter of the film and of the substrate. Therefore, if the dislocations relax effectively the mismatch deposition strain, they do not prevent the film to be tightly bound to the substrate and, therefore, the substrate imposes its thermal evolution to the film. This means that the dislocations do not relax the thermal strain that arises between the deposition temperature and room temperature. From Eq. 1, one gets:

$\frac{\mathrm{d} s_{\mathrm{m}}(T)}{\mathrm{d} T}=\left(\alpha_{b^{*}}-\alpha_{a_{0}}\right) \frac{a_{/ /}\left(T_{0}\right)}{a_{0}\left(T_{0}\right)} \cdot \frac{1}{\left(1+\alpha_{a_{0}} \Delta T\right)^{2}}$

From Eq. 2, it is clear that the temperature evolution of the misfit strain in the temperature-misfit strain phase diagrams are not vertical, not even linear strictly speaking. However, considering the fact that we deal with lead-based oxides, $\Delta T$ is limited because of the creation at high temperature of vacancies of volatile elements such as lead or oxygen. Considering that thermal expansion coefficients of usual substrates are in the range of $10^{-6}$ and $\Delta T \leq 10^{3}$, 
one sees that, at the first order, the evolution of the misfit strain with temperature is linear with a slope proportional to the difference in thermal expansion coefficients between the (effective) substrate and the bulk material. In the case of $\mathrm{Pb}\left(\mathrm{Zr}_{0.2} \mathrm{Ti}_{0.8}\right) \mathrm{O}_{3}$ thin films deposited on $\mathrm{SrTiO}_{3}$ [97]: $\mathrm{s}_{\mathrm{m}}$ $\left(T_{\mathrm{d}}=870 \mathrm{~K}\right)-\mathrm{s}_{\mathrm{m}}(R T) \simeq 0.3 \times 10^{-3}$ and for $\mathrm{PbTiO}_{3}$ thin film still on $\mathrm{SrTiO}_{3}$ [98]: $\mathrm{s}_{\mathrm{m}} \quad\left(T_{\mathrm{d}}=870 \mathrm{~K}\right)-$ $\mathrm{s}_{\mathrm{m}}(R T) \simeq-1.5 \times 10^{-3}$. These changes are of a smaller amplitude than the deposition strain but should nevertheless not be overlooked.

The third term of the misfit strain, after the deposition strain generated by the lattice mismatch and the thermal strain, is the spontaneous transformation strain, linked to the phase transition. In order to illustrate this, let's consider the hypothetical case of tetragonal $\mathrm{Pb}\left(\mathrm{Zr}_{0.2} \mathrm{Ti}_{0.8}\right) \mathrm{O}_{3}$ deposited on [001]-oriented $\mathrm{Pb}\left(\mathrm{Zr}_{0.2} \mathrm{Ti}_{0.8}\right) \mathrm{O}_{3}$. The substrate'surface presents a fourfold symmetry, potentially generating an isotropic biaxial strain state. The deposition of $\mathrm{Pb}\left(\mathrm{Zr}_{0.2} \mathrm{Ti}_{0.8}\right) \mathrm{O}_{3}$ in the optimal conditions would generate a coherent epitaxy (no misfit dislocations) and the film would be thick enough to neglect any finite size effect and thin enough to prevent the formation of domains. In that case, there is neither the strain due to the mismatch of the lattice parameters nor a thermal strain. The evolution of the misfit strain of this system with temperature is reported in Fig. 10. Its evolution in the paraelectric phase is linear, vertical, centered on zero. However, in the ferroelectric phase, the spontaneous strain associated to the cubic-tetragonal phase transition implies that the lattice parameters are not equal anymore to the pseudocubic one; the misfit strain is therefore not zero anymore and becomes negative: the symmetry of such a [001]-oriented $\mathrm{Pb}\left(\mathrm{Zr}_{0.2} \mathrm{Ti}_{0.8}\right) \mathrm{O}_{3}$ deposited on

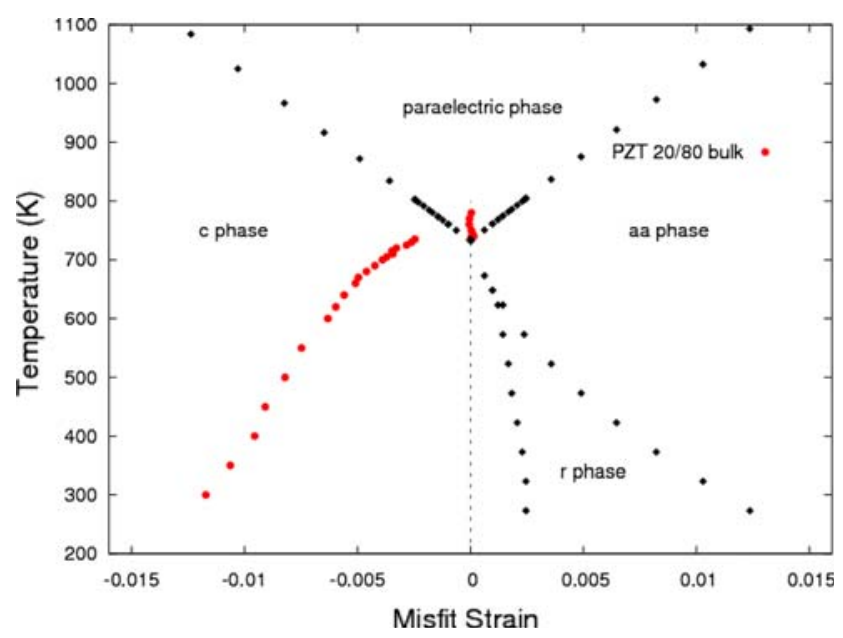

Fig. 10 Evolution with temperature of bulk $\mathrm{Pb}\left(\mathrm{Zr}_{0.2} \mathrm{Ti}_{0.8}\right) \mathrm{O}_{3}$ misfit strain: an hypothetical $\mathrm{Pb}\left(\mathrm{Zr}_{0.2} \mathrm{Ti}_{0.8}\right) \mathrm{O}_{3}$ thin film deposited on a [001]-oriented $\mathrm{Pb}\left(\mathrm{Zr}_{0.2} \mathrm{Ti}_{0.8}\right) \mathrm{O}_{3}$ substrate. The phase diagram of $\mathrm{Pb}\left(\mathrm{Zr}_{0.2} \mathrm{Ti}_{0.8}\right) \mathrm{O}_{3}$ has been calculated in [95] [001]-oriented $\mathrm{Pb}\left(\mathrm{Zr}_{0.2} \mathrm{Ti}_{0.8}\right) \mathrm{O}_{3}$ substrate is therefore, just like the bulk, tetragonal.

It is worth noting that the spontaneous strain is large. In the case of $100 \mathrm{~nm} \mathrm{~Pb}\left(\mathrm{Zr}_{0.2} \mathrm{Ti}_{0.8}\right) \mathrm{O}_{3}$ and $\mathrm{PbTiO}_{3}$ films deposited on $\mathrm{SrTiO}_{3}[97,98]$ it is of the same order of magnitude as the deposition strain, but of opposite sign, resulting in a near cancelation. It is then the thermal strain, despite its relative weakness, which imposes most of the misfit strain evolution [89]. This situation is however rather coincidental. Depositing in the bulk ferroelectric phase would be a way of canceling the influence of the spontaneous strain, depositing in the bulk paraelectric phase on a substrate matching the lattice parameter of the deposited material would be a way to annihilate the deposition strain and then the spontaneous strain would probably impose mainly the misfit strain. This underlines why the deposition conditions are of particular importance, not mentioning the defect-related questions.

\section{Misfit strain in the polydomain case}

In the polydomain case, the calculation of the misfit strain is complicated by the coexistence of multiple in-plane lattice parameters (see Fig. 11) and the dependence of the equilibrium fraction of c-domains on $s_{m}$ (cf. Eq. 2 in [95]). The complication arises from the necessity to measure several in-plane lattice parameters not only to have the precise value of the lattice parameters, but also to determine precisely the domain population. The misfit strain is given by [123]: $s_{\mathrm{m}}=\left(1-\phi_{\mathrm{c}}^{*}\right) \frac{a_{/ /}-a_{0}}{a_{0}}+\phi_{\mathrm{c}}^{*} \frac{c_{/ /}-a_{0}}{a_{0}}$.

\section{Clarification of the notations used in the isotropic biaxial stress field case}

Several articles have been published on the calculations of temperature-misfit strain phase diagrams and, unfortunately for the reader, the notations are not standardized and, in a few case, not appropriate. The aim of this paragraph is to review the notations used in the literature in the case of monodomain films submitted to an isotropic biaxial stress field, and to provide the reader with an equivalent in

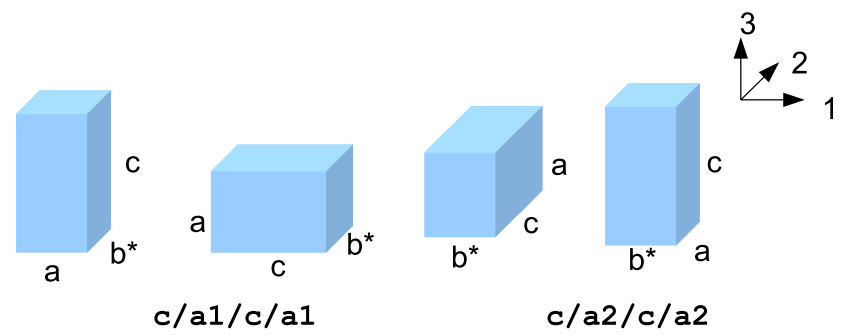

Fig. 11 Lattice parameters in the two possible $a / c / a / c$ domain structures 
Table 1 Isotropic biaxial stress field, monodomain phases: schematic representation, polarization's components, notations from Pertsev et al. [94] unless stated otherwise, symmetry, space group and basis vectors in the pseudo-cubic reference

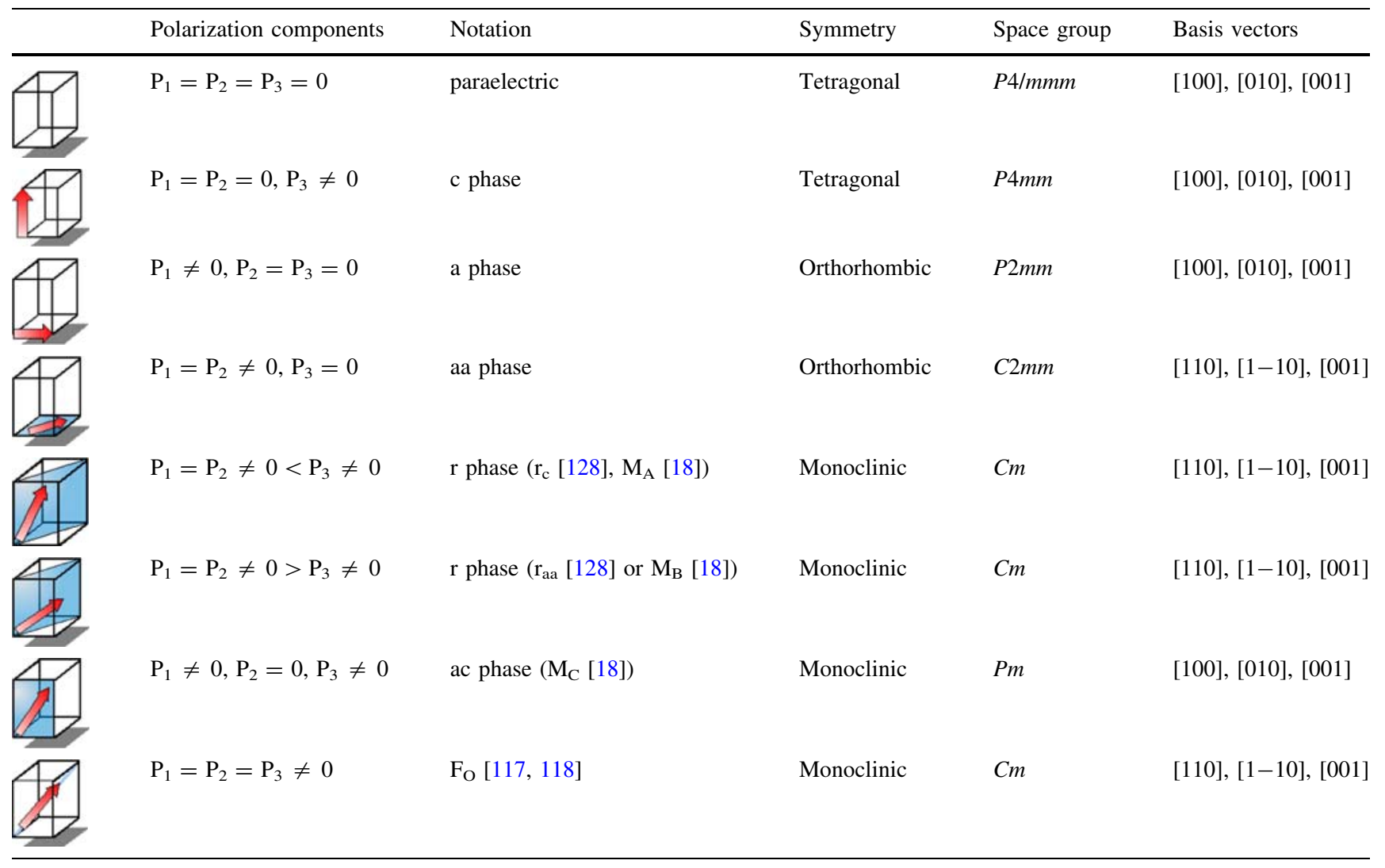

terms of crystallographic space groups. This is summarized in Table 1.

Most of the possible confusions come from the misuse of crystallographic notation to describe phases defined by the (non-)zero components of the polarization and by the confusion of the surface's (2D) symmetry and the crystal's (3D) one. We will take a few examples below.

Let us first consider the substrate. As mentioned before, in order to induce an isotropic biaxial stress field, a $<001>$-oriented cubic material $m$ may be used, as well as [001]-oriented tetragonal one. In both case, the relevant symmetry is the one of the surface i.e., the one of square. Using a $<011>$-oriented cubic substrate will result in anisotropic biaxial strain imposed on the film, as the symmetry of the surface will be the one of a rectangle.

Let us now review the possible phases, the associated polarization components and their crystallographic symmetries.

- In the paraelectric phase, a ferroelectric material behaves as a classical elastic material submitted to an isotropic biaxial strain. Therefore, the imposed in-plane deformation induces an out-of-plane deformation that can be calculated through Poisson's coefficient.
Therefore, the symmetry of the paraelectric phase is reduced from cubic in the bulk to tetragonal for thin films. It remains nevertheless centro-symmetric.

- In the c and aa phases, as the symmetry of the polarization mimics the one of the unit cell, the symmetries are generally adequately attributed to tetragonal and orthorhombic, respectively. However, the axes of the cell in the aa phase are rotated in-plane by $45^{\circ}$ with respect to the pseudo-cubic ones.

- The a phase, where $\mathrm{P}_{1} \neq 0, \mathrm{P}_{2}=\mathrm{P}_{3}=0$, is not tetragonal. Due to the displacement of the titanium along the [100] direction, the fourfold axis along [001] (i.e., the direction perpendicular to the interface) is lost. The symmetry of such a phase is then orthorhombic. This phase has never been reported for single domain temperature-misfit strain phase diagrams, probably as the two in-plane directions being equivalent, by symmetry, a "b" phase with $\mathrm{P}_{1}=0, \mathrm{P}_{2} \neq 0, \mathrm{P}_{3}=0$ would be as stable and would give rise to a polydomain ( " $\mathrm{a}_{1} /$ $\mathrm{a}_{2} / \mathrm{a}_{1} / \mathrm{a}_{2}$ ") structure (see "Polydomain films" section).

- The $r$ phase actually divides itself into two phases, depending on the relative magnitude of the in- and outof-plane components of the polarization. The $\mathrm{M}_{\mathrm{A}}$ and 
$M_{B}$ notations have been introduced [18] to make the distinction between these two cases. $\mathrm{M}_{\mathrm{A}}$ stands for $\mathrm{P}_{1}=\mathrm{P}_{2}>\mathrm{P}_{3}$ and $\mathrm{M}_{\mathrm{B}}$ for $\mathrm{P}_{1}=\mathrm{P}_{2}>\mathrm{P}_{3}$. As a side note, the Legendre transformation used to introduce the stress related terms in the Landau polynomial reduces the expansion order necessary to describe monoclinic symmetries. This symmetry can only be described by an eight order polynomial in the bulk whereas a six order is sufficient in thin films [18].

- The $M_{\mathrm{C}}$ notation was introduced [18] by analogy with the $\mathrm{M}_{\mathrm{A}}$ and $\mathrm{M}_{\mathrm{B}}$ ones, to stand for the $\mathrm{P}_{1} \neq 0, \mathrm{P}_{2}=0$, $\mathrm{P}_{3} \neq 0$ case (space group: $P m$ ). This ac phase has only been observed so far in a narrow region of $\mathrm{BaTiO}_{3}$ phase diagram (and noted either $\mathrm{M}_{1}^{\mathrm{F}}$ [129] or ac [94, 130]). This may be due to the fact that this symmetry implies a distinction between the two in-plane directions of the substrate.

- The "rhombohedral" case [117, 118] was considered with $\mathrm{P}_{1}=\mathrm{P}_{2}=\mathrm{P}_{3} \neq 0$ [117] or $\mathrm{P}_{1}=\mathrm{P}_{2} \neq 0$, $\mathrm{P}_{3} \neq 0, \mathrm{P}_{1} \neq \mathrm{P}_{3}$ [118]. This phase should actually be described as monoclinic with the latter definition and considered as the special case between the $\mathrm{M}_{\mathrm{A}}$ and $\mathrm{M}_{\mathrm{B}}$ monoclinic phases in the former one.

We hope that Table 1 will help the reader to adequately compare theoretical and experimental results, even though previous attempts $[18,105]$ to do so have unfortunately not prevented the use of erroneous terms, especially in the case of anisotropic biaxial stress fields as we shall see in the next paragraphs. Such a table will be given for the anisotropic biaxial stress field in "Clarification of the notations used in the anisotropic biaxial stress field case" section.

\section{Anisotropic 2D stress field}

A generalization of the concept of temperature-misfit strain phase diagrams considers the case of orthorhombic substrates. Such a substrate imposes two different strains in the plane of the interface as it has two distinct in-plane lattice parameters. Such a strain state has been called anisotropic or unequal biaxial in-plane/misfit/epitaxial strains. We shall use anisotropic biaxial misfit strain or stress field. As mentioned earlier, this encompasses also the case of [110]-oriented cubic substrates. Two methods have been used so far to address this problem: thermodynamics calculations and phase-field simulations. It has to be noted that no ab-initio based calculations have been carried out on these systems so far to the best of our knowledge.

This problem was first tackled considering monodomain systems. Then, polydomain systems started to be considered through phase-field simulations only. The main results are summarized below.

\section{Monodomain case}

The monodomain case is the simpler one to address and several misfit strain-misfit strain phase diagrams (at constant temperature) have been calculated by thermodynamic theory for $\mathrm{PbTiO}_{3}$ [131-135], $\mathrm{Pb}\left(\mathrm{Zr}_{0.5} \mathrm{Ti}_{0.5}\right) \mathrm{O}_{3}$ [134], $\left(\mathrm{Pb}_{0.35} \mathrm{Sr}_{0.65}\right) \mathrm{TiO}_{3}$ [131], $\mathrm{BaTiO}_{3}$ [132-134], $\left(\mathrm{Ba}_{x} \mathrm{Sr}_{1-x}\right)$ $\mathrm{TiO}_{3}(x=0.5$ [136], 0.7 [137]). Experimental reports such as the one about lead titanate thin films deposited on orthorhombic $\mathrm{DyScO}_{3}$ [138] showed that ac and $\mathrm{r}$ phases can be stabilized by anisotropic stress fields. One has to pay attention to the set of parameters used to calculate the phase diagrams as the use of an erroneous set leads to incorrect results (compare, for example, [134] and [132]).

The common conclusion is that anisotropic strain may stabilize phases that are absent from the monodomain isotropic strain case. The phase sequence for the isotropic case corresponds to the diagonal of the misfit strain-misfit strain diagrams and one can check that it remains unchanged, as expected. However, and as pointed out by Zembilgotov et al. [131], when the bulk paraelectric phase is cubic, the symmetry of the film's paraelectric phase is now centrosymmetric orthorhombic and not centrosymmetric tetragonal as in the case of isotropic strains.

However, experimentalists have to be cautious about the details of the calculations as the modified thermodynamics potential are often based on a sixth-order Landau-Devonshire development [131-133, 135-137] that limits both the temperature and the misfit-strain range on which the results are meaningful. See [134] and [139] in the case of $\mathrm{BaTiO}_{3}$, and [137] for $\mathrm{Ba}_{0.7} \mathrm{Sr}_{0.3} \mathrm{TiO}_{3}$. In addition, the use of an eight-order polynomial enables to address the question of the stability of the phase in which the three components of the polarization are not nil.

What is more, unless expressly addressed, the in-plane shear strain ( $\mathrm{u}_{6}$ in Voigt's notation) is taken nil. Taking into account this strain destabilizes the phase where $\mathbf{P}=\left(\mathrm{P}_{1}=\right.$ $0, \mathrm{P}_{2}=0, \mathrm{P}_{3} \neq 0$ ) [134], leading to a shift of the $180^{\circ}$ ferroelectric domain walls compared to the hypothetical case where the film would be free from the substrate and therefore free to be strained in the $\mathrm{z}$ direction [140]. In addition, the use of an eight-order Landau polynomial leads to the complete destabilization, from a thermodynamic point of view, of the phase in which all components are not nil $\left(\mathbf{P}=\left(\mathrm{P}_{1} \neq 0, \mathrm{P}_{2} \neq 0, \mathrm{P}_{3} \neq 0\right)\right)$.

The application of an external uniaxial mechanical loading on the surface of a thin film was studied in the isotropic case by Pertsev et al. [141]. Thin films grown on orthorhombic substrates and submitted to such external loads show a tendency to stabilize the paraelectric phase and therefore enable a stress-driven phase transition between ferro- and paraelectric states [133]. However, this study was restricted to the case where the two in-plane 
strains are equal in magnitude but of opposite sign $\left(\mathrm{u}_{m 1}=-\mathrm{u}_{m 2}\right)$.

The influence of depolarizing fields, the other key player in the determination of the structure of ferroelectric thin films, has been considered recently on 8-nm thin film [135]. Uncompensated charges at the surface/interface of a ferroelectric thin film as well as a gradient of polarization throughout the film generate a depolarizing field. This field can be partially screened by the deposition of electrodes, resulting in a situation, between open-circuit conditions (no charge compensation, maximum depolarizing field) and short-circuit conditions (complete compensation, depolarizing field only generated by the polarization gradient). From a first-principle point of view, the partial screening of the depolarizing field has been modeled through a $\beta$ coefficient [142]. Under ideal open-circuit conditions, $\beta=0$ the depolarizing field is therefore maximal, whereas for a given $\beta=\beta_{\mathrm{SC}}$ the depolarizing field vanishes. Quite naturally, the depolarizing field destabilizes the phase in which $\mathbf{P}=\left(\mathrm{P}_{1}=0, \mathrm{P}_{2}=0, \mathrm{P}_{3} \neq 0\right)$ and stabilizes the phases with non-zero in-plane components of the polarization [135]. This first result is similar to what happens under isotropic misfit strain [128]. In addition, depolarizing fields stabilize phases exhibiting only one non-zero inplane component of the polarization $\left(\mathbf{P}=\left(\mathrm{P}_{1} \neq 0, \mathrm{P}_{2}=\right.\right.$ $\left.0, \mathrm{P}_{3}=0\right)$ or $\left.\mathbf{P}=-\left(\mathrm{P}_{1}=0, \mathrm{P}_{2} \neq 0, \mathrm{P}_{3}=0\right)\right)$ in detrimental to the phases with both in- and out-of-plane nonzero components of the polarization, which were stable without any depolarizing field. It is worth noting that a partial screening of the surface charges has a pronounced effect only when the partial compensation of the charges rises above 99\% [135]. This seems to indicate than under realistic conditions (i.e., with surface charges partially screened) the real system is closer to the open-circuit conditions than the short-circuit ones.

The consequences on dielectric properties of films [131, 134, 136, 137], their tunability [137] and pyroelectric properties [136, 137] of such strain conditions have been calculated. These comparisons between the phenomenological predictions and the experimental measurements may lead to reasonable agreement as long as sufficient expansion of the Landau polynomial, appropriate electrical boundary conditions, available and reliable set of parameters have been used in the model and, as importantly, accurate measurement of the lattice parameters have been carried out [134].

Clarification of the notations used in the anisotropic biaxial stress field case

As for the isotropic strain state, much confusion arises from the use of crystallographic terms to describe phases that are in fact defined by the components of polarization. Table 2 presents the symmetry of the phases one may encounter in misfit strain-misfit strain diagrams.

A phase, where $\mathbf{P}=\left(\mathrm{P}_{1} \neq 0, \mathrm{P}_{2}=0, \mathrm{P}_{3}=0\right)$, is not tetragonal as claimed by several authors [133, 136, 137] (not even under an isotropic strain state, as we saw in "Clarification of the notations used in the isotropic biaxial stress field case" section) but orthorhombic. In addition, the phases with two non-zero polarization components are monoclinic, as the polarization is confined within one of the $<001>$-planes, and not orthorhombic [133, 136, 143]. Finally, the phase with $\mathrm{P}_{1} \neq 0, \mathrm{P}_{2} \neq 0$, and $\mathrm{P}_{3} \neq 0$ is triclinic (as pointed out by Zembilgotov et al. [131]) and not rhombohedral [143].

An appropriate description of the crystalline symmetry of the calculated phases would ease comparisons between experimental and theoretical results and therefore be beneficial for the whole community.

\section{Polydomain case}

In parallel to thermodynamical calculations, phase-field simulations have been carried out very recently in order to calculate temperature-misfit strain phase diagrams under anisotropic biaxial strain fields as well as the misfit strainmisfit strain diagrams at constant temperature. The cases of $\mathrm{PbTiO}_{3}$ [144, 145] and $\mathrm{BaTiO}_{3}$ [143] have been considered. In the phase-field simulations, the considered energy is the sum of the bulk free energy, the elastic deformation energy, the domain wall energy, and the electrostatic energy. As for thermodynamic calculations, the order of the expansion of the Landau polynomial has to be carefully checked before comparing either experimental results with theoretical predictions or between the phase diagrams.

\section{Domain structures}

Anisotropic biaxial strain increases the complexity of the possible domain structures in comparison with the isotropic case described before. As in the monodomain case, the isotropic misfit strain situation corresponds to the diagonal of the misfit strain-misfit strain phase diagrams. The possible domain structures are the following in the anisotropic case:

- $\mathrm{a}_{1}$ and $\mathrm{a}_{2}$ domains are not equivalent anymore and therefore the $\mathrm{a} / \mathrm{c} / \mathrm{a} / \mathrm{c}$ structure splits into $\mathrm{a}_{1} / \mathrm{c}\left(\right.$ or $\left.\mathrm{a}_{1}+\mathrm{c}\right)$ and $\mathrm{a}_{2} / \mathrm{c}$ (or $\left.\mathrm{a}_{2}+\mathrm{c}\right)$ structures.

- the $\mathrm{a}_{1}$ monodomain structure can now be stabilized thanks to the anisotropy of the strain. The same is true for the $a_{2}$ monodomain structure. These two monodomain structures are not stable in the isotropic case [87].

- the $\mathrm{a}_{1} / \mathrm{a}_{2} / \mathrm{c}$ (or $\mathrm{a}_{1}+\mathrm{a}_{2}+\mathrm{c}$ ) structure can also be stabilized by anisotropic misfit strains, whereas only $a_{i} / c / a_{i} / c$ phases were stable in the isotropic case. 
Table 2 Anisotropic biaxial stress field, monodomain phases: Schematic representation, polarization's components, notations, symmetry, space group, and basis vectors in the pseudo-cubic reference

\begin{tabular}{|c|c|c|c|c|}
\hline Polarization components & Notation & Symmetry & Space group & Basis vectors \\
\hline $\mathrm{P}_{1}=\mathrm{P}_{2}=\mathrm{P}_{3}=0$ & Paraelectric & Orthorhombic & $P 2 / \mathrm{mmm}$ & {$[100],[010],[001]$} \\
\hline $\mathrm{P}_{1}=\mathrm{P}_{2}=0, \mathrm{P}_{3} \neq 0$ & $\mathrm{c}[131,132,133,143]$ phase & Orthorhombic & $P 2 m m$ & {$[100],[010],[001]$} \\
\hline $\mathrm{P}_{1} \neq 0, \mathrm{P}_{2}=\mathrm{P}_{3}=0$ & $a^{\prime}[131,133], a_{1}[143,132]$ phase & Orthorhombic & $P 2 m m$ & {$[100],[010],[001]$} \\
\hline $\mathrm{P}_{1}=0, \mathrm{P}_{2} \neq 0, \mathrm{P}_{3}=0$ & $a^{\prime \prime}[131,133], a_{2}[143,132]$ phase & Orthorhombic & $P 2 \mathrm{~mm}$ & {$[100],[010],[001]$} \\
\hline $\mathrm{P}_{1} \neq 0, \mathrm{P}_{2} \neq 0, \mathrm{P}_{3}=0$ & $\mathrm{aa}^{*}$ [131], $\mathrm{a}^{\prime} \mathrm{a}^{\prime \prime}$ [133], $\mathrm{O}_{12}$ [143], $\mathrm{a}_{1} \mathrm{a}_{2}$ [132] phase & Monoclinic & $P m$ & [100], [010], [001] \\
\hline $\mathrm{P}_{1} \neq 0, \mathrm{P}_{2}=0, \mathrm{P}_{3} \neq 0$ & $\mathrm{a}^{\prime} \mathrm{c}[131,133], \mathrm{O}_{13}[143], \mathrm{a}_{1} \mathrm{c}$ [132] phase & Monoclinic & $\mathrm{Pm}$ & {$[100],[010],[001]$} \\
\hline $\mathrm{P}_{1}=0, \mathrm{P}_{2} \neq 0, \mathrm{P}_{3} \neq 0$ & $a^{\prime \prime} c[131,133], O_{23}[143], a_{2} c[132]$ phase & Monoclinic & $P m$ & {$[100],[010],[001]$} \\
\hline $\mathrm{P}_{1} \neq 0, \mathrm{P}_{2} \neq 0, \mathrm{P}_{3} \neq 0$ & $\mathrm{r}^{*}[131], \gamma^{\prime}[133], \mathrm{r}$ [143] phase & Triclinic & $P 1$ & {$[100],[010],[001]$} \\
\hline
\end{tabular}

- the c domains adopt the orthorhombic symmetry instead of the tetragonal one in the isotropic misfit strain case.

- the a domains, however, remain orthorhombic.

Temperature-misfit strain phase diagrams for polydomain structures under anisotropic biaxial stress field

$\mathrm{PbTiO}_{3}$ In the case of lead titanate, the domain structure was calculated using short-circuit boundary conditions with a six-order Landau polynomial [144]. The resulting polydomain phase diagram differs significantly from the single domain one [131]. The main difference with the monodomain misfit strain-misfit strain diagram lies into the destabilization of the phase with three non-zero polarization components in favor of a polydomain structure in which the domains with an out-of-plane component of the polarization coexist with those having one of the in-plane ones non-zero. In addition, the regions of stability of the monodomain phases with only one non-zero in-plane component $\left(\mathbf{P}=\left(\mathrm{P}_{1}, 0,0\right)\right.$ or $\left.\mathbf{P}=\left(0, \mathrm{P}_{2}, 0\right)\right)$ is pushed back to extremely asymmetric and large strains.

$\mathrm{BaTiO}_{3}$ Even though $\mathrm{BaTiO}_{3}$ does not belong to PZT phase diagram, it is the material on which a comparative study of the thermodynamics calculations and phase field simulations have been carried out [143]. It, therefore, enables the direct comparison between the two techniques and the resulting monodomain misfit strain-misfit strain diagrams from thermodynamics (eight-order Landau polynomial) and polydomain misfit strain-misfit strain from phase-field simulations (short-circuit boundary conditions).

\section{Influence of thickness}

Thickness is obviously an important parameter in thin film. In many phenomenological as well as ab initio-based models, thickness is not considered and the strain is 
supposed to be homogeneous throughout the whole thickness of the film. The thickness effect is peculiar to the thin film system, i.e., including the substrate, as it was reported that very thin slabs (down to $75 \mathrm{~nm}$ ) of bulk ferroelectrics $\left(\mathrm{BaTiO}_{3}\right)$ exhibit bulk-like properties, refuting therefore the thickness- or electrode-related nature of both the change of the order of the phase transition and the smearing of the associated dielectric peak [103].

Thickness can modify the strain state of the film through a gradient of deformation along the growth direction, it also defines whether a polydomain state is energetically favored compared to a monodomain one; the question of a minimum thickness for the onset of ferroelectricity is still debated and thickness plays a crucial role in the generation of dislocations. Therefore, several critical thicknesses can be met in the literature and the following paragraphs deal with them.

\section{Thickness dependent relaxation}

Qiu et al. have published recently a thickness versus misfit strain phase diagram for $\mathrm{PbTiO}_{3}$ thin films from thermodynamical calculations [146]. This study considers the possibility of stabilizing monodomain structures and generating misfit dislocations with realistic electrodes (considered through their screening length in the expression of the depolarizing field). They find that the monoclinic socalled $r$ phase is stable on very narrow ranges of misfit strain and thickness and that the tetragonal $c$ phase or the polydomain a/c/a/c phase are the most probable ones considering typical experimental conditions. Their phase diagrams also exhibit the possibility to stabilize a new phase, called $a^{\prime} c^{\prime}$, in which the polarization components are equal to the ones of the $\mathrm{c}$ and a phase, i.e., $\mathrm{P}^{a^{\prime} c^{\prime}}{ }_{[100]}=\mathrm{P}_{[100]}^{a}$ and $\mathrm{P}^{a^{\prime} c^{\prime}}{ }_{[001]}=\mathrm{P}^{c}{ }_{[001]}$. This phase diagram incorporates all the main parameters determining the stable phase. It is however based on the Matthews-Blakeslee criteria which underestimates the critical thickness for dislocation generation (see "Critical thickness for dislocations generation" section) and therefore the absolute value of the mentioned thicknesses should be taken with care.

An exponential profile over the thickness for the stress has been considered in several models [147] and compared to experimental variation with thickness of the lattice parameters for $\mathrm{Pb}\left(\mathrm{Zr}_{0.4} \mathrm{Ti}_{0.6}\right) \mathrm{O}_{3}$ [147]. Catalan et al. [148] have used this exponential decay of the strain and X-ray diffraction analysis to calculate the strain profile. They have then introduced this gradient into a phenomenological calculation and calculated the dielectric constant as a function of both temperature and thickness, to finally compare their calculations with their experiments. This shows that strain gradient, through the flexoelectric effect can alter the dielectric properties of thin films even when their thickness is of several hundreds of nanometers [148].

A different behavior was reported recently by Bartasyte et al. on multidomain $\mathrm{PbTiO}_{3}$ thin films (from 30 to $460 \mathrm{~nm}$ thick) on $\mathrm{LaAlO}_{3}$ [149]. Stress measurements were carried out by X-ray diffraction and Raman spectroscopy, leading to residual stresses between 0.4 and $2 \mathrm{GPa}$ that are in the range of reported values for polycrystalline thin films (see [149] and references therein) and comparable to the ones reported for monodomain thin films [97, 98]. The evolution of the a domain fraction presents a maximum whereas the residual stress decreases linearly. This is contradictory with the temperature-misfit strain phase diagrams proposed in [96]. Indeed, at constant temperature, the domain population is a monotonous function (but not continuous at the transition between the $\mathrm{a} / \mathrm{c} / \mathrm{a} / \mathrm{c}$ and $\mathrm{a}$ phases according to the domain stability map, see "Domain stability map" section) of the misfit strain. However, considering the room temperature thickness-misfit strain phase diagram developed by Qiu et al. [146] and forgetting about the absolute values of the thickness reported on it, one sees that the domain population evolves in a non linear way in the $\mathrm{c} / \mathrm{a} / \mathrm{c} / \mathrm{a}$ phase. From the out-of-plane lattice parameters reported by Bartasyte et al. [149] and considering an elastic deformation of a tetragonal material characterized by a Poisson coefficient of 0.37 [89], one finds that the (thermodynamic) misfit strain goes from approximatively 0 (for the thickest film) to $+2 \%$ (for the thinnest one). Therefore, a non-linear evolution of the domain population with thickness is not in contradiction with this phase diagram. For clarity sake, let us note that the "misfit strain" evoked in [149] is due to the lattice mismatch between the substrate and the bulk and their "residual stress" is linked to the actual lattice parameter of the film, and therefore to the (thermodynamic) misfit strain. These two are markedly different, as pointed out by Bartasyte et al. as they are of opposite signs.

A surprising result has been reported by Gariglio et al. [101] on monodomain tetragonal $\mathrm{Pb}\left(\mathrm{Zr}_{0.2} \mathrm{Ti}_{0.8}\right) \mathrm{O}_{3}$ thin films deposited on ( $\mathrm{Nb}$ doped) $\mathrm{SrTiO}_{3}$ substrates. Even though elastic relaxation occurs when thickness is increased from 15 to $123 \mathrm{~nm}$ (through the exponential increase of the in-plane lattice parameter and the corresponding decrease of the out-of-plane one) the transition temperature remains constant. This report is in contradiction with other reports by Fong et al. on $\mathrm{PbTiO}_{3}$ ultrathin films (from 3 to 30 unit cells) deposited on $\mathrm{SrTiO}_{3}$ [150] or $\mathrm{SrRuO}_{3}$ [151]. As pointed out by the authors, this seems in contradiction with the temperature-misfit strain phase diagram developed by Pertsev et al. [94]. However, the value of the misfit strain for the thinnest film has been calculated by the authors to be of $-23 \times 10^{-3}$ [101], 
which is not in the range of the misfit strain considered in the phase diagram.

Let us recall that the Landau polynomial used by Pertsev et al. [94] is of sixth order and therefore limits the range of the misfit strains that can be considered. As the misfit strain for the thickest film is still of $9 \times 10^{-3}$ it lies out of the range of misfit reported on Pertsev's et al. phase diagram [94].

Critical thickness for the onset of ferroelectricity

Due to the cooperative nature of ferroelectricity, the question of the minimum thickness in which ferroelectricity can develop is of particular interest. Ferroelectricity has been measured in $5 \mathrm{~nm}$ [152], $4 \mathrm{~nm}$ [153] and even in a $1.2 \mathrm{~nm}[150,154,155]$ crystalline films and in a $1 \mathrm{~nm}$ polymer [156] film. Taking into account realistic interface between the ferroelectric thin film and the electrodes, i.e., a finite screening length of the electrodes as well as the details of the interface, ab-initio calculations showed that a critical thickness for ferroelectricity still exist [157]. Despite the short circuit conditions imposed on the electrodes, a depolarizing field arises into the film due to the imperfect screening of the surface charges by the electrodes.

Recently, Glinchuk et al. [158] showed, thanks to phenomenological calculations, that the reduction of thickness under nonideal short-circuit conditions leads not to a paraelectric state but to an electret state. This state is characterized by the vanishing of the polarization loop, the variation of the polarization with applied electric field being asymmetrical and shifted by the internal electric field that develops due to the imperfect screening of the surface charges. In the electret state pyroelectric effect remains and the film does not transit to a nonpolar state at higher temperature. A critical thickness under which the film is paraelectric only exists when no internal field exist (under the application of a canceling exterior electric field for example) [158].

Very recently, Sai et al. [159] studied by first-principles ultra-thin improper ferroelectric films, i.e., materials for which the primary order parameter, the one driving the phase transition, is not the polarization. They show for isolated single domain $\mathrm{YMnO}_{3}$ ultrathin films (down to 2 unit cell thick) that there is no critical thickness under which the paraelectric state is stable, even under open circuit boundary conditions.

Critical thickness for mono- to polydomain transition

With increasing thickness, a transition from a monodomain state (i.e., possibly containing only $180^{\circ}$ domains in the tetragonal case) to a polydomain state (with $90^{\circ}$ domains in the same symmetry) has been considered theoretically [85$88,91]$. In a monodomain film, the $180^{\circ}$ domains reduce the depolarizing field, forming domain stripes as observed on $\mathrm{PbTiO}_{3}$ thin films [73]. In a polydomain film, the $90^{\circ}$ domain structure is a consequence of the minimization of elastic energies as well as the electrostatic one. The concept of critical thickness for domain formation in ferroelectric thin film was developed by Pompe et al. [88]. In the same article, the thickness dependence of the minimum domain wall thickness was also developed. Their model considered only the competition between elastic energy reduction and increase in energy due to domain wall creation. Electrical conditions, kinetics of domain wall motion, energy linked to defect generation, nucleation of domains were assumed to be negligible. Although these apparent limitations, their calculations of the critical thickness were confirmed on $30 \mathrm{~nm} \mathrm{PbTiO}_{3}$ thin films deposited on $\mathrm{DyScO}_{3}$ [160] where a critical thickness around $10 \mathrm{~nm}$ and a domain structure consisting of alternating wide $\mathrm{c}$ domains and narrow a domains (with width down to the minimum value of 6-7 nm) with a period of $27-31 \mathrm{~nm}$. These values are the minimum ones preserving the horizontal coherency of the film. This configuration does not fall into the scope of the theory developed by Roytburd et al. [85-87] and Pertsev et al. [123] as the domain periodicity has to be smaller than the thickness of the film. Considering Kittel's law [161], which states that the domain period increases with the square root of the thickness of the film, one sees that Roytburd's model implies to consider thicker film than the one studied by Vlooswijk et al. [160].

The misorientation of the different variants of the tetragonal domains illustrated in Fig. 1 implies to take a closer look at the situation near the interface with the surface. Instead of parallel domain walls, steps have been observed by transmission electron microscopy [71] in the domain wall profile leading to wedge-shaped a domains. This was later confirmed by calculations [162]. In addition, the domains were observed to start from dislocations standing off the interface [71]. This shape minimizes the strain at the interface due to the clamping on the substrate and enables the misorientation to be compensated. This shape is however a function of the stiffness of the substrate, a higher stiffness leading to a higher interface strain and therefore minimizing the width of the a domain a the interface with substrate [71, 162]. As a consequence, the a domain walls observed on the $\mathrm{SrRuO}_{3}$ electrode deposited on $\mathrm{DyScO}_{3}$ were parallel [71]. It has to be noted that domains were intentionally generated in these $\mathrm{PbTiO}_{3}$ films deposited on $\mathrm{SrTiO}_{3}$ by modifying the deposition conditions. For tetragonal $\mathrm{PbZrTiO}_{3}$ films grown under optimal conditions (i.e., as close as possible to the thermodynamic equilibrium), the expected and observed domain structure 
consists of $\left(180^{\circ}\right) \mathrm{c}$ domains, in this thickness range (for $\mathrm{PbTiO}_{3}: 100 \mathrm{~nm}$ in [98], $110 \mathrm{~nm}$ in [71]; for $\mathrm{Pb}$ $\left(\mathrm{Zr}_{0.2} \mathrm{Ti}_{0.8}\right) \mathrm{O}_{3}: 90 \mathrm{~nm}$ in [163], $100 \mathrm{~nm}$ in [97]).

\section{Critical thickness for dislocations generation}

The difference in thermal expansion coefficients does not give rise to a thermal strain that is sufficient to enable dislocation generation during cooling from the deposition temperature (on the contrary to what was considered [111]). This is confirmed by the thermal evolution of the in-plane lattice parameters of monodomain films that run parallel to the substrate's one $[97,98,101,100]$. Additional dislocations would indeed enable the in-plane lattice parameter of the film to relax toward its bulk value. The dislocations are therefore introduced during deposition or at the bulk para-ferroelectric transition.

Frank and van der Merwe [164, 165] introduced 60 years ago the concept of the critical thickness above which misfit dislocation formation was the energetically favored stress-relieving mechanism at the interface of an heteroepitaxial system. More precisely, considering a monolayer, they introduced the notion of critical misfit value above which introducing dislocation was energetically favored. The Matthews-Blakeslee [166] model has been the most used one to calculate this critical thickness. It is based on equilibrium considerations with its equations derived from the search of the total (i.e., elastic strain and dislocation) energy minimum or from equilibrating the forces acting on a dislocation.

From Matthews-Blakeslee's criteria [166], the linear density of dislocation $(\rho)$ is expected to vary according to:

$\rho|\mathbf{b}| \cos (\lambda)=\epsilon_{\mathrm{m}}-\frac{l(h)}{(1+v) h}$

with $|\mathbf{b}|$ the norm of Burgers vector, $\lambda$ the angle between the Burgers vector, and a line belonging to both the interface and to a plane normal to the dislocation line, $\epsilon_{m}=\frac{a_{/ /}-a_{0}}{a_{0}}$ the misfit strain, $h$ the thickness, $v$ Poisson coefficient. The last term, $l(h)$, is defined as: $l(h)=$ $\frac{|\mathbf{b}|}{8 \pi \cos \lambda}\left(1-v(\cos \beta)^{2}\right) \ln \left(\frac{\alpha h}{|\mathbf{b}|}\right)$ with $\beta$ the angle between the dislocation line and the Burgers vector, and $\alpha$ a parameter taking into account the nature of the chemical bonding (varying between three for ionic crystals and four for covalent ones). In the case of a monodomain film as well as in the $a_{1} / a_{2} / a_{1} / a_{2}$ structure, we would like to repeat that the Burgers vectors lie in the plane of the interface and the domain walls are vertical (see "Limitations" section). Therefore $\mathbf{b} / /[100]$ or [010] $\left(|\mathbf{b}|=\max \left(a_{\mathrm{s}}, a_{/ /}\right)\right), \lambda=0$, and $\beta=\pi / 2$.

Following Speck and Pompe [111], this dislocation density could be approximated by: $\rho \approx \frac{\epsilon_{\mathrm{m}}}{|\mathbf{b}|(\cos (\lambda))^{2}}\left(1-\frac{t_{\mathrm{cr}}}{t}\right)$

However this implies, as pointed out in their paper: $l(h) \approx l\left(h_{\mathrm{c}}\right)$, i.e., $\ln h \approx \ln h_{\mathrm{c}}$.

This model proved to be adequate to describe the situation in metals. Several models have been developed from Matthews-Blakeslee's, for metals and semiconductors. Let us cite the one by Fischer et al. [167] who extended Matthews-Blakeslee's model to take into account the interaction between straight dislocations and the one by Chidambarrao et al. [168] who also extended it by taking into account the effect of Peierls barrier. However, for ferroelectrics, it usually predicts critical thicknesses that are smaller than what has been observed experimentally.

Another model, developed by People and Bean [169] for semiconductors, considered a nucleation barrier that had to be overcome by the misfit strain in order to favor misfit dislocations generation. Venkatesan et al. [71] used this latter model to describe their $\mathrm{PbTiO}_{3}$ thin films deposited on $\mathrm{SrTiO}_{3}$. The thickness range covered by their study extends from 22 to $340 \mathrm{~nm}$ and a slow growth rate prevented the formation of a domains. High-resolution transmission electron microscopy and X-ray diffraction revealed the absence of dislocations for all thickness up to $340 \mathrm{~nm}$. The critical thickness derived from People and Bean's model reads $[169,170]$ :

$t_{\mathrm{cr}}=\frac{1-v}{1+v} \frac{1}{16 \pi \sqrt{2}} \frac{|\mathbf{b}|^{2}}{a_{0}} \frac{1}{\epsilon^{2}} \ln \left(\frac{t_{\mathrm{cr}}}{|\mathbf{b}|}\right)$

with $\varepsilon$ the relative difference between the substrate and bulk lattice parameters. Venkatesan et al. considered the expression of People and Bean's model reported by Maree et al. [171] for the case plotted by People and Bean in [169] (and corrected in [170]) in which they considered $|\mathbf{b}| \approx$ $2 \sqrt{2} a_{0}$ and considered five $<110>$ atom spacings, hence the slightly different expression reported by Maree et al. and used by Venkatesan et al. Interestingly, following People and Bean's model, calculations have shown that the use of small seed pads on a substrate enable the critical thickness to tend toward infinity for pads with lateral dimensions below a critical size [172]. Even if the orientation of the Burgers vector and the mean value of the effective interfacial width of isolated dislocations may be different from the one considered, the work by Venkatesan et al. points out meaningfully the discrepancies in critical thickness that can be calculated depending on the model considered.

Their work underlines therefore the need for a model designed specifically for ferroelectrics or, more generally for functional oxides, which is still lacking despite the numerous experimental evidence of the influence of dislocations on the properties of ferroelectric thin films. 
Jesser and Fox [173] showed that on substrates not as perfect as silicon ones may be, the propagation of preexisting dislocations (rather than a nucleation barrier) was the limiting parameter. Indeed, this friction stress is much higher in semiconductors than in metals. In addition, the density of dislocations generated on these not-so-perfect substrates is evidently much higher and therefore their interaction may come into play. They then developed [173] a model including frictional stress applicable to both metals and semiconductors.

A comprehensive study of the defect microstructure in tetragonal $\mathrm{Pb}\left(\mathrm{Zr}_{0.2} \mathrm{Ti}_{0.8}\right) \mathrm{O}_{3}$ thin films on $\mathrm{SrTiO}_{3}$ has been reported by Misirlioglu et al. [174]. The investigated thin films were mainly c-oriented with a minority of $\mathrm{a}_{1}$ and $\mathrm{a}_{2}$ domains and displayed both threading dislocations as well as misfit dislocations. The density of the former ones is high (over $10^{10} \mathrm{~cm}^{-2}$ ) and their origin is attributed, in a VolmerWeber growth mode, to the coalescence of the islands, forcing the misfit dislocations away from the interface with the substrate [174]. The former explanation of threading dislocations being the continuation of the ones existing in the substrate [166] could only account for a small fraction of them. Misirlioglu et al. pointed out [174] that the domain structure, created to accommodate part of the spontaneous strain associated with the phase transition, nucleates at misfit dislocations. These domains are then pinned because of the microstresses generated by the misfit dislocations and therefore the extrinsic contribution (i.e., domain wall movements) to the piezoelectric coefficients that plays a major role in the bulk is strongly reduced in thin films. This lead the authors to conclude that the electrical and electromechanical properties of polydomain thin films should be inferior to the bulk ones. Thermodynamic calculations further showed that the impact of threading dislocations (with a direction line perpendicular to the interface) was "limited" to the smearing of the transition temperature, and that the strongest impact on the other functional properties was indeed due to the misfit dislocations [174].

This is somehow in contradiction with the measurements of $180^{\circ}$ domain wall creeplike velocity in tetragonal PZT films [175, 176], complemented by measurements of domain wall roughness [177]. For $180^{\circ}$ domain walls other models describe their motion, such as the dynamic poling model developed by Trolier-McKinstry et al. [178]. However, the clamping of $90^{\circ}$ domain walls was shown to be reduced through nanoisland patterning of the film using focused ion beam, resulting in a strong enhancement of the properties [179]. It is worth mentioning that, in addition to dislocations, point defects, such as oxygen vacancies, have a critical influence on the domain wall thickness [180].

The misfit dislocations, through the localized strain field generated around them, induce locally a strong reduction of polarization which is detrimental to the properties of the film (see [174] and references therein for the consequences on polarization loops, dielectric, and piezoelectric properties). This was confirmed recently by the study of polarization and piezoelectric properties on $\mathrm{Pb}\left(\mathrm{Zr}_{0.52} \mathrm{Ti}_{0.48}\right) \mathrm{O}_{3}$ thin films [110]. The strong reduction of polarization was also shown recently to reach $48 \%$ in a $\mathrm{Pb}\left(\mathrm{Zr}_{0.2} \mathrm{Ti}_{0.8}\right) \mathrm{O}_{3}$ film deposited on $\mathrm{SrTiO}_{3}$ and covered by a $\mathrm{SrTiO}_{3}$ film [181]. Morelli et al. [182] attributed this effect to the steps observed in the domain walls [71] giving rise to leakage currents that dominate the polarization reversal measured by piezo-force miscroscopy. Interfacial dislocations were also held responsible for the poor piezoresponse observed for $\mathrm{Pb}\left(\mathrm{Zr}_{0.52} \mathrm{Ti}_{0.48}\right) \mathrm{O}_{3}$ nanoislands [183]. A comparison between the properties of $\mathrm{Pb}\left(\mathrm{Zr}_{0.2} \mathrm{Ti}_{0.8}\right) \mathrm{O}_{3}$ films free from extended defects and films presenting $90^{\circ}$ domains have been carried out by Vrejoiu et al. [163]. If the polarization is indeed reduced in the case of defective films, the dielectric constant was found to be higher, indicating that the pinning of the domain walls by misfit dislocations is not as effective as to suppress completely extrinsic contributions to the dielectric properties of ferroelectric thin films as predicted in [174]. A recent review of both the influence of growth conditions and of the microstructure of perovskite films has been published recently by Vrejoiu et al. [184].

Pálová et al. [185] have proposed a model drastically different from the ones mentioned so far. Instead of a strain gradient induced by defects present throughout the whole film, a segregated strain gradient is proposed. This model is shown to reproduce the observed thickness dependence of ferroelectric thin films in a comparable manner as the ones based on inhomogeneous strains. In Pálová et al.'s model, the defects are located at the interface with the substrate, over a given thickness. As a consequence, the internal bias field generated is constant throughout the film (for a given thickness), whereas the bias field created by inhomogeneous strains in the film varies within the film. Hence, an applied electric field should be able to cancel the bias field of the segregated model and the film should exhibit bulklike properties. This is the benchtop probe proposed by Pálová et al. [185] that remains to be tested to the best of our knowledge.

\section{Future developments}

In this brief section, we provide a few suggestions for possible directions of future developments.

Anisotropic misfit strain

The misfit strain-misfit strain diagrams enable the experimentalists to compare the structure of the film with the 
calculations or simulations at room temperature. However, in order to compare the evolution with temperature of the film's lattice parameters, more temperature-dependent results would be of great interest. In addition, no firstprinciple based calculations results have been published so far on anisotropic biaxial misfit strain phase diagrams. The influence of different growth directions has indeed already been considered [186], but the strain field applied on the material was taken as isotropic. These calculations, though computer-wise intensive, would provide the community with predictive tools for reduced-size device design.

\section{Rotation of the oxygen octahedra}

A usual mechanism of accommodation of strain in the bulk is oxygen octahedra rotation. As pointed out in the introduction, this has been observed and calculated both for low temperature and high pressure. This mechanism has only been taken into account so far in calculations on $\mathrm{SrTiO}_{3}$ [141, 187], despite its potential importance in all perovskites.

Determination of the precise extrapolation length

Taking into account the electrical boundary condition in the calculations requires the experimentalists to measure it with great precision.

Strain profile throughout the thickness

So far thickness dependent properties relied on series of films with different thickness. The measurement of the actual stress profile within a single film should enable to study the influence of the (top) surface on this profile. As was shown experimentally in $\mathrm{PbTiO}_{3}$ ultrathin films on $\mathrm{SrTiO}_{3}$ by Fong et al. [188], the displacement of atoms and the resulting unit cell can evolve in a way much more complex than the simple exponential. The usual tetragonality-polarization direct relation as well as the tetragonality throughout the film was shown by high-resolution transmission electron microscopy to vary through ultrathin $(7 \mathrm{~nm})$ tetragonal films [189] in a way not easily compatible with an exponential decay. A better understanding and description of this strain gradient would therefore be of great interest, including to design novel device based on properties such as the flexoelectric effect.

Open Access This article is distributed under the terms of the Creative Commons Attribution Noncommercial License which permits any noncommercial use, distribution, and reproduction in any medium, provided the original author(s) and source are credited.

\section{References}

1. Setter N, Damjanovic D, Eng L, Fox G, Gevorgian S, Hong S, Kingon A, Kohlstedt H, Park NY, Stephenson GB, Stolitchnov I, Taganstev AK, Taylor DV, Yamada T, Streiffer S (2006) J Appl Phys 100(5):051606. doi:10.1063/1.2336999. http://link.aip.org/ link/?JAP/100/051606/1

2. Dawber M, Rabe KM, Scott JF (2005) Rev Mod Phys 77(4): 1083

3. Scott J, Morrison FD, Lou MMZPX, Kugler VM, Rios S, Zhang M, Tatsuta T, Tsuji O, Leedham TJ (2005) J Am Ceram Soc 88(7):1691. doi:10.1111/j.1551-2916.2005.00486.x

4. Kingon A, Streiffer SK (1999) Curr Opin Solid State Mater Sci 4(1):39

5. Setter N (2005) Electroceramic based MEMS. Springer, New York

6. Ramesh R (1997) Thin film ferroelectric materials and devices. Kluwer Academic, Dordrecht

7. Paz de Araujo C, Scott JF, Taylor GW (1996) Ferroelectric thin films: synthesis and basic properties. Gordon and Breach, Amsterdam

8. Jaffe B, Cook W, Jaffe H (1971) Piezoelectric ceramics. Academic, London

9. Shirane G, Takeda A (1952) J Phys Soc Jpn 7(1):5. doi: 10.1143/JPSJ.7.5. http://jpsj.ipap.jp/link?JPSJ/7/5/

10. Noheda B, Cox D, Shirane G, Gonzalo J, Cross L, Park SE (1999) Appl Phys Lett 74(14):2059

11. Noheda B, Gonzalo J, Cross L, Guo R, Park SE, Cox D, Shirane G (2000) Phys Rev B 61(13):8687

12. Noheda B, Cox D, Shirane G, Guo R, Jones B, Cross L (2000) Phys Rev B 63:014103

13. Kornev IA, Bellaiche L, Janolin PE, Dkhil B, Suard E (2006) Phys Rev Lett 97:157601. doi:10.1103/PhysRevLett.97.157601

14. Hatch D, Stokes H, Ranjan R, Ragini, Mishra S, Pandey D, Kennedy B (2002) Phys Rev B 65:212101

15. Ranjan R, Singh AK, Ragini, Pandey D (2005) Phys Rev B 71(9):092101

16. Singh AK, Pandey D (2001) J Phys Condens Matter 13(48):931

17. Singh AK, Pandey D (2003) Phys Rev B (Condens Matter Mater Phys) 67(6):064102

18. Vanderbilt D, Cohen M (2001) Phys Rev B 63(094108):094108

19. Fu H, Cohen RE (2000) Nature 403(67):281

20. Bellaiche L, Garcia A, Vanderbilt D (2000) Phys Rev Lett 84(23):5427

21. Noheda B, Cox D (2006) Phase Trans 79(1-2):5. http://www. informaworld.com/10.1080/01411590500467262

22. Grinberg I, Cooper VR, Rappe AM, Nature 419(6910n):909. doi:10.1038/nature01115

23. Bell A (2006) J Mater Sci 41(1):13. doi:10.1007/s10853-0055913-9

24. Sergienko I, Gufan Y, Urazhdin S (2002) Phys Rev B 65:144104

25. Singh AK, Pandey D, Yoon S, Baik S, Shin N (2007) Appl Phys Lett 91(19):192904. doi:10.1063/1.2804008. http://link.aip.org/ link/?APL/91/192904/1

26. Singh AK, Mishra SK, Ragini, Pandey D, Yoon S, Baik S, Shin N (2008) Appl Phys Lett 92(2):022910. doi:10.1063/1.2836269. http://link.aip.org/link/?APL/92/022910/1

27. Pandey D, Singh AK, Baik S (2008) Acta Crystallogr Sect A 64(1):192. doi:10.1107/S0108767307055511

28. Wang YU (2006) Phys Rev B (Condens Matter Mater Phys) 74(10):104109. doi:10.1103/PhysRevB.74.104109. http://link. aps.org/abstract/PRB/v74/e104109

29. Wang YU (2007) Phys Rev B (Condens Matter Mater Phys) 76(2):024108. doi:10.1103/PhysRevB.76.024108. http://link.aps. org/abstract/PRB/v76/e024108 
30. Schierholz R, Fuess H, Tsuda K, Ogata Y, Terauchi M, Theissmann R (2008) Phys Rev B (Condens Matter Mater Phys) 78(2):024118. doi:10.1103/PhysRevB.78.024118. http://link.aps. org/abstract/PRB/v78/e024118

31. Mishra SK, Pandey D, Singh AP, Appl Phys Lett 69(12):1707. doi:10.1063/1.118004. http://link.aip.org/link/?APL/69/1707/1

32. Almahmoud E, Kornev I, Bellaiche L (2009) Phys Rev Lett 102(10):105701. doi:10.1103/PhysRevLett.102.105701. http://link. aps.org/abstract/PRL/v102/e105701

33. Roberts S (1950) J Am Ceram Soc 33(2):63. doi:10.1111/ j.1151-2916.1950.tb14168.x

34. Shirane G, Sawaguchi E, Takeda A (1950) Phys Rev 80(3):485

35. Sawaguchi E, Shirane G, Takagi Y (1951) J Phys Soc Jpn 6(5):333. doi:10.1143/JPSJ.6.333. http://jpsj.ipap.jp/link?JPSJ/ $6 / 333 /$

36. Shirane G, Sawaguchi E, Takagi Y (1951) Phys Rev 84(3):476. doi:10.1103/PhysRev.84.476

37. Roberts S (1951) Phys Rev 83(5):1078. doi:10.1103/PhysRev. 83.1078 .2

38. Sawaguchi E, Maniwa H, Hoshino S (1951) Phys Rev 83(5):1078. doi:10.1103/PhysRev.83.1078

39. Shirane G, Suzuki K (1952) J Phys Soc Jpn 7(3):333. doi: 10.1143/JPSJ.7.333. http://jpsj.ipap.jp/link?JPSJ/7/333/

40. Jona F, Shirane G, Mazzi F, Pepinsky R (1957) Phys Rev 105(3):849. doi:10.1103/PhysRev.105.849

41. Tennery VJ (1965) J Electrochem Soc 112(11):1117. doi: 10.1149/1.2423374. http://link.aip.org/link/?JES/112/1117/1

42. Tennery VJ (1966) J Am Ceram Soc 49(9):483. doi:10.1111/ j.1151-2916.1966.tb13304.x

43. Scott B, Burns G (1972) J Am Ceram Soc 55(7):331. doi: 10.1111/j.1151-2916.1972.tb11303.x.

44. Kittel C (1951) Phys Rev 82(5):729. doi:10.1103/PhysRev. 82.729

45. Whatmore RW, Glazer AM (1979) J Phys C Solid State Phys 12(8):1505. http://stacks.iop.org/0022-3719/12/1505

46. Fesenko O, Kolesova R, Sindeyev Y (1978) Ferroelectrics 20:177

47. Singh DJ (1995) Phys Rev B 52(17):12559. doi:10.1103/Phys RevB.52.12559

48. Sawaguchi E, J Phys Soc Jpn 8(5):615. doi:10.1143/JPSJ.8.615. http://jpsj.ipap.jp/link?JPSJ/8/615/

49. Suchanicz J, Kania A (2008) Phase Trans 81:1089. http://www. informaworld.com/10.1080/01411590802460791

50. Fritz IJ (1978) J Appl Phys 49(9):4922. doi:10.1063/1.325527. http://link.aip.org/link/?JAP/49/4922/1

51. Berlincourt D, Krueger HHA (1959) J Appl Phys 30(11):1804. doi:10.1063/1.1735059. http://link.aip.org/link/?JAP/30/1804/1

52. Budimir M, Damjanovic D, Setter N (2005) Phys Rev B 72(6):064107. doi:10.1103/PhysRevB.72.064107

53. Duan Y, Shi H, Qin L (2008) J Phys Condens Matter 20(17): 175210 (5 p). http://stacks.iop.org/0953-8984/20/175210

54. Janolin PE, Dkhil B, Davis M, Damjanovic D, Setter N (2007) Appl Phys Lett 90(15):152907. doi:10.1063/1.2721856. http:// link.aip.org/link/?APL/90/152907/1

55. Rapoport E (1966) Phys Rev Lett 17(21):1097. doi:10.1103/ PhysRevLett.17.1097

56. Samara GA (1970) Phys Rev B 1(9):3777. doi:10.1103/Phys RevB.1.3777

57. Handerek J, Pisarski M, Ujma Z (1981) J Phys C Solid State Phys 14(14):2007. http://stacks.iop.org/0022-3719/14/2007

58. Furuta H, Endo S, Ming LC, Kobayashi M (1999) Phys B Condens Matter 263-264:816. doi:10.1016/S0921-4526(98) 01464-1. http://www.sciencedirect.com/science/article/B6TVH3W9KY78-80 /2/5b8d8d885af401e2d829621dce1af1f9

59. Kornev IA, Bellaiche L, Bouvier P, Janolin PE, Dkhil B, Kreisel J (2005) Phys Rev Lett 95(19):196804
60. Janolin PE, Bouvier P, Kreisel J, Thomas PA, Kornev IA, Bellaiche L, Crichton W, Hanfland M, Dkhil B (2008b) Phys Rev Lett 101(23):237601. doi:10.1103/PhysRevLett.101.237601. http://link.aps.org/abstract/PRL/v101/e237601

61. Ahart M, Somayazulu M, Cohen RE, Ganesh P, Dera P, Mao Hk, Hemley RJ, Ren Y, Liermann P, Wu Z (2008) Nature 451(7178):545. doi:10.1038/nature06459

62. Ganesh P, Cohen RE (2009) J Phys Condens Matter 21(6): 064225 (4 p). http://stacks.iop.org/0953-8984/21/064225

63. Frantti J, Fujioka Y, Nieminen RM (2008) J Phys Condens Matter 20(47):472203 (4 p). http://stacks.iop.org/0953-8984/20/ 472203

64. Yamamoto T, Makino Y (1996) Jpn J Appl Phys 35(Part 1, No. 5B):3214. doi:10.1143/JJAP.35.3214. http://jjap.ipap.jp/link?JJAP/ 35/3214/

65. Rouquette J, Haines J, Bornand V, Pintard M, Papet P, Bousquet C, Konczewicz L, Gorelli F, Hull S (2004) Phys Rev B 70:014108

66. Rouquette J, Haines J, Bornand V, Pintard M, Papet P, Marshall WG, Hull S (2005) Phys Rev B (Condens Matter Mater Phys) 71(2):024112

67. Rouquette J, Haines J, Fraysse G, Al-Zein A, Bornand V, Pintard M, Papet P, Hull S, Gorelli FA (2008) Inorg Chem 47(21):9898. doi:10.1021/ic8008688. http://pubs.acs.org/doi/abs/ $10.1021 / \mathrm{ic} 8008688$

68. Rouquette J, Haines J, Pintard M, Papet P, Bonnet B, Gorelli F (2003) Solid State Sci 5:451

69. Wu Z, Cohen RE (2005) Phys Rev Lett 95(3):037601

70. Frantti J, Fujioka Y, Nieminen RM (2007) J Phys Chem B 111(17):4287. doi:10.1021/jp0713209. http://pubs.acs.org/doi/ abs/10.1021/jp0713209

71. Venkatesan S, Kooi BJ, Hosson JTMD, Vlooswijk AHG, Noheda B (2007) J Appl Phys 102(10):104105. doi:10.1063/ 1.2815657. http://link.aip.org/link/?JAP/102/104105/1

72. Kornev I, Clausen M, Lai B, Naumov I, Ponomareva I, Fu H, Bellaiche L (2008) Handbook of advanced dielectric, piezoelectric and ferroelectric materials. Synthesis, properties and applications. Woodhead Publishing Limited, Cambridge, England. http://www.woodheadpublishing.com/en/book.aspx?bookID=1256. ISBN 184569186 5, ISBN-13: 9781845691868

73. Streiffer S, Eastman J, Fong D, Thompson C, Munkholm A, Murty MR, Auciello O, Bai G, Stephenson G (2002) Phys Rev Lett 89(6):067601

74. Lee KS, Baik S (1999) J Appl Phys 85(3):1995

75. Janolin PE, Fraisse B, Dkhil B, Marrec FL, Ringgaard E, Appl Phys Lett 90(16):162906. doi:10.1063/1.2727563. http://link. aip.org/link/?APL/90/162906/1

76. Hsu WY, Raj R (1995) Appl Phys Lett 67(6):792

77. Ramesh R, Sands T, Keramidas VG (1993) Appl Phys Lett 63(6):731

78. Roemer A, Millon E, Vincent B, Boudrioua A, Pons-Y-Moll O, Defourneau RM, Seiler W (2004) J Appl Phys 95(6):3041

79. Lee KS, Baik S (2000) J Appl Phys 87(11):8035

80. Kwak BS, Erbil A, Budai JD, Chisholm MF, Boatner LA, Wilkens BJ (1994) Phys Rev B 49(21):14865. doi:10.1103/ PhysRevB.49.14865

81. Nagarajan V, Jenkins I, Alpay S, Li H, Aggarwal S, SamalancaRiba L, Roytburd A, Ramesh R (1999) J Appl Phys 86(1):595

82. Batzer RS, Yen BM, Liu D, Chen H, Kubo H, Bai GR (1996) J Appl Phys 80(11):6235

83. Kim S, Kang Y, Baik S (1996) J Am Ceram Soc 79(4):1105

84. Pertsev NA, Zembilgotov AG (1996) J Appl Phys 80(11):6401

85. Roytburd A (1999) J Appl Phys 83(1):239

86. Roytburd AL (1989) J Appl Phys 83(1):228. doi:10.1063/ 1.366677. http://link.aip.org/link/?JAP/83/228/1

87. Alpay S, Roytburd A (1998) J Appl Phys 83(9):4714 
88. Pompe W, Gong X, Suo Z, Speck J (1993) J Appl Phys 74(10): 6012

89. Janolin PE (2006) Instabilites structurales sous contraintes dans les materiaux ferroelectriques. Ph.D. thesis, Ecole Centrale Paris

90. Kukhar VG, Pertsev NA, Kohlstedt H, Waser R (2006) Phys Rev B (Condens Matter Mater Phys) 73(21):214103. doi: 10.1103/PhysRevB.73.214103. http://link.aps.org/abstract/PRB/ v73/e214103

91. Foster CM, Pompe W, Daykin AC, Speck JS (1996) J Appl Phys 79(3):1405. doi:10.1063/1.360978. http://link.aip.org/link/?JAP/ $79 / 1405 / 1$

92. Alpay SP, Nagarajan V, Bendersky LA, Vaudin MD, Aggarwal S, Ramesh R, Roytburd AL (1999) J Appl Phys 85(6):3271

93. Ederer C, Spaldin NA (2005) Phys Rev Lett 95(25):257601. doi: 10.1103/PhysRevLett.95.257601

94. Pertsev N, Zembilgotov A, Tagantsev A (1988) Phys Rev Lett 80(9): 1988

95. Pertsev NA, Kukhar VG, Kohlstedt H, Waser R (2003) Phys Rev B 67(5):054107. doi:10.1103/PhysRevB.67.054107

96. Koukhar V, Pertsev N, Waser R (2001) Phys Rev B 64:214103

97. Janolin PE, Fraisse B, Marrec FL, Dkhil B (2007) Appl Phys Lett 90(21):212904. doi:10.1063/1.2742313. http://link.aip.org/ link/?APL/90/212904/1

98. Janolin PE, Marrec FL, Chevreul J, Dkhil B (2007) Appl Phys Lett 90(19):192910. doi:10.1063/1.2738060. http://link.aip.org/ link/?APL/90/192910/1

99. Bartasyte A, Dkhil B, Kreisel J, Chevreul J, Chaix-Pluchery O, Rapenne-Homand L, Jimenez C, Abrutis A, Weiss F (2008) Appl Phys Lett 93(24):242907. doi:10.1063/1.3046787. http:// link.aip.org/link/?APL/93/242907/1

100. Venkatesan S, Vlooswijk A, Kooi BJ, Morelli A, Palasantzas G, Hosson JTMD, Noheda B (2008) Phys Rev B (Condens Matter Mater Phys) 78(10):104112. doi:10.1103/PhysRevB.78.104112. http://link.aps.org/abstract/PRB/v78/e104112

101. Gariglio S, Stucki N, Triscone JM, Triscone G, Appl Phys Lett 90(20):202905. doi:10.1063/1.2740171. http://link.aip.org/link/ ?APL/90/202905/1

102. Lee KS, Kang YM, Baik S (1999) J Mater Res 14(1):132

103. Saad MM, Baxter P, Bowman RM, Gregg JM, Morrison FD, Scott JF, J Phys Condens Matter 16(41):L451. http://stacks.iop. org/0953-8984/16/L451

104. Ghosez P, Junquera J, Handbook of theoretical and computational nanotechnology, vol 9. American Scientific Publishers, Stevenson Ranch

105. Kornev I, Fu H, Bellaiche L (2006) J Mater Sci 41(1):137. doi: 10.1007/s10853-005-5962-0

106. Speck JS, Seifert A, Pompe W, Ramesh R (1994) J Appl Phys 76(1):477. doi:10.1063/1.357098. http://link.aip.org/link/?JAP/ $76 / 477 / 1$

107. Misirlioglu IB, Vasiliev A, Aindow M, Alpay SP (2005) Integr Ferroelectr 71:67

108. Suzuki T, Nishi Y, Fujimoro M (1999) Philos Mag A 79(10): 2461

109. Canedy C, Li H, Alpay S, Salamanca-Riba L, Roytburd A, Ramesh R (2000) Appl Phys Lett 77(11):1695

110. Nagarajan V, Jia CL, Kohlstedt H, Waser R, Misirlioglu IB, Alpay SP, Ramesh R (2005) Appl Phys Lett 86(19):192910

111. Speck J, Pompe W (1994) J Appl Phys 76(1):466

112. Doukhan N, Doukhan JC (1986) Phys Chem Mineral 13(6):403. doi:10.1007/BF00309185

113. Wang Y, Poirier JP, Liebermann RC (1989) Phys Chem Mineral 16(7):630. doi:10.1007/BF00223310

114. Kawasaki M, Takahashi K, Maeda T, Tsuchiya R, Shinohara M, Ishiyama O, Yonezawa T, Yoshimoto M, Koinuma H (1994) Science 266(5190):1540. doi:10.1126/science.266.5190.1540. http://www.sciencemag.org/cgi/content/abstract/266/5190/1540
115. Ohnishi T, Shibuya K, Lippmaa M, Kobayashi D, Kumigashira H, Oshima M, Koinuma H (2004) Appl Phys Lett 85(2):272. doi:10.1063/1.1771461. http://link.aip.org/link/?APL/85/272/1

116. Nagarajan V, Ganpule CS, Li H, Salamanca-Riba L, Roytburd AL, Williams ED, Ramesh R (2001) Appl Phys Lett 79(17):2805

117. Oh SH, Jang HM (1998) Appl Phys Lett 72(12):1457

118. Oh SH, Jang HM (2000) Phys Rev B 62(22):14757

119. Morioka H, Yokoyama S, Oikawa T, Funakubo H, Saito K (2004) Appl Phys Lett 85(16):3516

120. Roytburd AL (1976) Physica Status Solidi (a) 37(1):329

121. Kim YK, Morioka H, Ueno R, Yokoyama S, Funakubo H, Lee K, Baik S (2006) Appl Phys Lett 88(25):252904

122. Roytburd AL, Alpay SP, Bendersky LA, Nagarajan V, Ramesh R (2001) J Appl Phys 89(1):553. doi:10.1063/1.1328781. http://link.aip.org/link/?JAP/89/553/1

123. Pertsev N, Koukhar V (2000) Phys Rev Lett 84(16):3722

124. Cava RJ, Takagi H, Krajewski JJ, Peck WF, Hwang HY (1993) Phys Rev B 47(17):11525

125. Buban J, Iddir H, Ogut S (2004) Phys Rev B 69:108102

126. Hünnefeld H, Niemöller T, Schneider J, Rntt U, Rodewald S, Fleig J, Shirane G (2002) Phys Rev B 66:014113

127. Lee JW, Park GT, Park CS, Kim HE (2006) Appl Phys Lett 88(7):072908

128. Lai BK, Kornev I, Bellaiche L, Salamo G (2005) Appl Phys Lett $86: 132904$

129. Li YL, Chen LQ (2006) Appl Phys Lett 88(7):072905

130. Pertsev NA, Zembilgotov ZG, Tagantsev AK (1999) Ferroelectrics 223(1-4):79

131. Zembilgotov AG, Pertsev NA, Bottger U, Waser R (2005) Appl Phys Lett 86(5):052903. doi:10.1063/1.1855389. http://link.aip. org/link/?APL/86/052903/1

132. Wang J, Zhang TY (2005) Appl Phys Lett 86(19):192905. doi: 10.1063/1.1923765. http://link.aip.org/link/?APL/86/192905/1

133. Qiu JH, Jiang Q (2007) J Appl Phys 101(3):034110. doi: 10.1063/1.2433135. http://link.aip.org/link/?JAP/101/034110/1

134. Zembilgotov AG, Bottger U, Waser R (2008) J Appl Phys 104(5):054118. doi:10.1063/1.2976347. http://link.aip.org/link/ ?JAP/104/054118/1

135. Wang J, Zhang TY (2008) Phys Rev B (Condens Matter Mater Phys) 77(1):014104. doi:10.1103/PhysRevB.77.014104. http:// link.aps.org/abstract/PRB/v77/e014104

136. Tao YM, Wu YZ (2007) J Appl Phys 101(2):024111. doi: 10.1063/1.2430642. http://link.aip.org/link/?JAP/101/024111/1

137. Cao HX, Lo VC, Li ZY (2007) J Appl Phys 101(1):014113. doi: 10.1063/1.2407272. http://link.aip.org/link/?JAP/101/014113/1

138. Catalan G, Janssens A, Rispens G, Csiszar S, Seeck O, Rijnders G, Blank DHA, Noheda B (2006) Phys Rev Lett 96(12):127602

139. Li YL, Cross LE, Chen LQ (2005) J Appl Phys 98(6):064101. doi: 10.1063/1.2042528. http://link.aip.org/link/?JAP/98/064101/1

140. Bratkovsky, Levanyuk AP (2001) Phys Rev Lett 86(16):3642. doi:10.1103/PhysRevLett.86.3642

141. Pertsev N, Tagantsev A, Setter N (2002) Phys Rev B 65:219901

142. Kornev I, Fu H, Bellaiche L (2004) Phys Rev Lett 93(19): 196104. doi:10.1103/PhysRevLett.93.196104

143. Sheng G, Zhang JX, Li YL, Choudhury S, Jia QX, Liu ZK, Chen LQ (2008) Appl Phys Lett 93(23):232904. doi:10.1063/ 1.3039410. http://link.aip.org/link/?APL/93/232904/1

144. Sheng G, Zhang JX, Li YL, Choudhury S, Jia QX, Liu ZK, Chen LQ (2008) J Appl Phys 104(5):054105. doi:10.1063/1.2974093. http://link.aip.org/link/?JAP/104/054105/1

145. Liu B, Pidugu S, Bhattacharyya A (2008) Phys Rev B (Condens Matter Mater Phys) 77(2):024102. doi:10.1103/PhysRevB.77. 024102. http://link.aps.org/abstract/PRB/v77/e024102

146. Qiu QY, Nagarajan V, Alpay (2008) Phys Rev B (Condens Matter Mater Phys) 78(6):064117. doi:10.1103/PhysRevB. 78.064117. http://link.aps.org/abstract/PRB/v78/e064117 
147. Kim HJ, Oh SH, Jang HM (1999) Appl Phys Lett 75(20):3195. doi:10.1063/1.125275. http://link.aip.org/link/?APL/75/3195/1

148. Catalan G, Noheda B, McAneney J, Sinnamon LJ, Gregg JM (2005) Phys Rev B 72(2):020102

149. Bartasyte A, Chaix-Pluchery O, Kreisel J, Jimenez C, Weiss F, Abrutis A, Saltyte Z, Boudard M (2008) J Appl Phys 103(1): 014103. doi:10.1063/1.2821728. http://link.aip.org/link/?JAP/103/ 014103/1

150. Fong D, Stephenson G, Streiffer S, Eastman J, Auciello O, Fuoss P, Thompson C (2004) Science 304:1650

151. Fong DD, Kolpak AM, Eastman JA, Streiffer SK, Fuoss PH, Stephenson GB, Thompson C, Kim DM, Choi KJ, Eom CB, Grinberg I, Rappe AM, Phys Rev Lett 96(12):127601. doi: 10.1103/PhysRevLett.96.127601. http://link.aps.org/abstract/PRL/ v96/e127601

152. Lichtensteiger C, Triscone JM, Junquera J, Ghosez P (2005) Phys Rev Lett 94(4):047603. doi:10.1103/PhysRevLett.94.04 7603. http://link.aps.org/abstract/PRL/v94/e047603

153. Tybell T, Ahn C, Triscone JM (1999) Appl Phys Lett 75(6):856

154. Spaldin N (2004) Science 304:1606

155. Despont L, Koitzsch C, Clerc F, Garnier MG, Aebi P, Lichtensteiger C, Triscone JM, de Abajo FJG, Bousquet E, Ghosez P (2006) Phys Rev B 73(9):094110

156. Ducharme S, Fridkin VM, Bune AV, Palto SP, Blinov LM, Petukhova NN, Yudin SG (2000) Phys Rev Lett 84(1):175. doi: 10.1103/PhysRevLett.84.175

157. Junquera J, Ghosez P (2003) Nature 422:506

158. Glinchuk MD, Morozovska AN, Eliseev EA (2006) J Appl Phys 99(11):114102. doi:10.1063/1.2198940. http://link.aip.org/link/ ?JAP/99/114102/1

159. Sai N, Fennie CJ, Demkov AA (2009) Phys Rev Lett 102(10):107601. doi:10.1103/PhysRevLett.102.107601. http:// link.aps.org/abstract/PRL/v102/e107601

160. Vlooswijk AHG, Noheda B, Catalan G, Janssens A, Barcones B, Rijnders G, Blank DHA, Venkatesan S, Kooi B, de Hosson JTM (2007) Appl Phys Lett 91(11):112901. doi:10.1063/1.2783274. http://link.aip.org/link/?APL/91/112901/1

161. Kittel C (1949) Rev Mod Phys 21(4):541. doi:10.1103/RevMod Phys. 21.541

162. Li YL, Hu SY, Liu ZK, Chen LQ (2002) Acta Mater 50(2):395. doi:10.1016/S1359-6454(01)00360-3. http://www.sciencedirect. com/science/article/B6TW8-44V0RH6-G/2/f5196cce2e24dcf1f $6966 \mathrm{e} 6745176197$

163. Vrejoiu I, LeRhun G, Pintilie L, Hesse D, Alexe M, Gsele U (2006) Adv Mater 18(13):1657. doi:10.1002/adma.200502711

164. Frank FC, van der Merwe JH (1949) Proc Roy Soc Lond Ser A Math Phys Sci 198(1053):205. doi:10.1098/rspa.1949.0095. http:// rspa.royalsocietypublishing.org/content/198/1053/205.abstract

165. Frank FC, van der Merwe JH (1949) Proc R Soc Lond Ser A Math Phys Sci 198(1053):216. doi:10.1098/rspa.1949.0096. http:// www.rspa.royalsocietypublishing.org/content/198/1053/216. abstract

166. Matthews J, Blakeslee A (1974) J Cryst Growth 27:118

167. Fischer A, Kühne H, Richter H (1994) Phys Rev Lett 73(20): 2712. doi:10.1103/PhysRevLett.73.2712
168. Chidambarrao D, Srinivasan GR, Cunningham B, Murthy CS (1990) Appl Phys Lett 57(10):1001. doi:10.1063/1.103548. http://link.aip.org/link/?APL/57/1001/1

169. People R, Bean JC (1985) Appl Phys Lett 47(3):322. doi: 10.1063/1.96206. http://link.aip.org/link/?APL/47/322/1

170. People R, Bean JC (1986) Appl Phys Lett 49(4):229. doi: 10.1063/1.97637. http://link.aip.org/link/?APL/49/229/1

171. Maree PMJ, Barbour JC, van der Veen JF, Kavanagh KL, BulleLieuwma CWT, Viegers MPA (1987) J Appl Phys 62(11):4413. doi:10.1063/1.339078. http://link.aip.org/link/?JAP/62/4413/1

172. Luryi S, Suhir E (1986) Appl Phys Lett 49(3):140. doi: 10.1063/1.97204. http://link.aip.org/link/?APL/49/140/1

173. Jesser W, Fox B (1990) J Electr Mater 19(11):1289. doi: 10.1007/BF02673344

174. Misirlioglu I, Vasiliev A, Alpay S, Aindow M, Ramesh R (2006) J Mater Sci 41(3):697. doi:10.1007/s10853-006-6488-9

175. Tybell T, Paruch P, Giamarchi T, Triscone JM (2002) Phys Rev Lett. 89(9):097601

176. Paruch P, Triscone JM (2006) Appl Phys Lett 88(16):162907

177. Paruch P, Giamarchi T, Triscone JM (2005) Phys Rev Lett 94(19): 197601

178. Trolier-McKinstry S, Gharb NB, Damjanovic D (2006) Appl Phys Lett 88(20):202901. doi:10.1063/1.2203750. http://link. aip.org/link/?APL/88/202901/1

179. Nagarajan V, Roytburd A, Stanishevsky A, Prasertchoung S, Zhao T, Chen L, Melngailis J, Auciello O, Ramesh R (2003) Nat Mater 2(1):43. doi:10.1038/nmat800

180. Shilo D, Ravichandran G, Bhattacharya K (2004) Nat Mater 3(7):453. doi:10.1038/nmat 1151

181. Jia CL, Mi SB, Urban K, Vrejoiu I, Alexe M, Hesse D (2009) Phys Rev Lett 102(11):117601. doi:10.1103/PhysRevLett.102. 117601. http://link.aps.org/abstract/PRL/v102/e117601

182. Morelli A, Venkatesan S, Palasantzas G, Kooi BJ, Hosson JTMD (2007) J Appl Phys 102(8):084103. doi:10.1063/1.27 94859. http://link.aip.org/link/?JAP/102/084103/1

183. Chu MW, Szafraniak I, Scholz R, Harnagea C, Hesse D, Alexe M, Gosele U Nat Mater 3(2):87. doi:10.1038/nmat1057

184. Vrejoiu I, Alexe M, Hesse D, Gsele U (2008) Adv Funct Mater 18(24):3892. doi:10.1002/adfm.200800560

185. Pálová L, Chandra P, Rabe KM (2007) Phys Rev B (Condens Matter Mater Phys) 76(1):014112. doi:10.1103/PhysRevB.76. 014112. http://link.aps.org/abstract/PRB/v76/e014112

186. Ponomareva I, Bellaiche L (2006) Phys Rev B (Condens Matter Mater Phys) 74(6):064102. doi:10.1103/PhysRevB.74.064102. http://link.aps.org/abstract/PRB/v74/e064102

187. Pertsev N, Tagantsev A, Setter N (2000) Phys Rev B 61(2):R825

188. Fong DD, Cionca C, Yacoby Y, Stephenson GB, Eastman JA, Fuoss PH, Streiffer SK, Thompson C, Clarke R, Pindak R, Stern EA (2005) Phys Rev B 71(14): 144112

189. Jia CL, Nagarajan V, He JQ, Houben L, Zhao T, Ramesh R, Urban K, Waser R (2007) Nat Mater 6(1):64. doi:10.1038/ nmat 1808 